Concurrence en contrats, anti-sélection

et structure d'information

\author{
Marie-Cécile Fagart ${ }^{1} 2$
}

$n^{\circ} 9317$

${ }^{1}$ Maître de Conférences à lUniversité de Paris X, Cepremap.

2 Je remercie Bruno Jullien pour son aide. 


\title{
Concurrence en contrats, anti-sélection et structure d'information
}

Résumé:

On s'intéresse dans cet article à un jeu entre deux principaux et un agent, quand l'agent détient une information de façon privée. On montre que le jeu de concurrence en contrats que se livrent les deux principaux admet un équilibre efficace au sens du premier rang, quand le gain que tire chaque principal de l'échange est indépendant du type de l'agent. Sous cette hypothèse, la concurrence en contrat n'a donc pas les effets soulignés par Rotschild et Stiglitz (1976), à savoir inefficacité et inexistence. Une explication en terme de précision de structure d'information est proposée.

Mots Clés: Incitations, concurrence, anti-sélection

Classement JEL: D 82

Competition between principals, adverse selection and information schemes

\begin{abstract}
:
This paper is dealing with a game where two principals compete for an agent, and when the agent has private information. When the payoff of the principal does not depend on private information, the studied game has an efficient equilibrium. Competition in markets with asymetric information does not always imply loss of efficiency, as suggested by Rotschild and Stiglitz (1976). An explain in terms of type of uncertainty is proposed.
\end{abstract}

key words: incentives, competition, aderse selection.

JEL classification numbers: D 82 
L'étude de la relations contractuelle entre deux agents quand l'échange auquel ils se livrent est affecté par des problèmes d'asymétrie d'information et lorsque cet échange est soumis à des pressions concurrentielles a reçu beaucoup de développements depuis l'article pionnier de Rothschild et Stiglitz (1976).

Rappelons que ces auteurs s'intéressent aux problèmes d'asymétrie d'information et de concurrence sur un marché d'assurance. Des compagnies d'assurance offrent des contrats à des particuliers, dont elles ignorent le risque d'accident, représenté par la probabilité qu'a chaque agent de subir un dommage. Les agents, quant à eux connaissent parfaitement leurs probabilités individuelles d'avoir un accident. Lorsque la concurrence est suffisamment forte, hypothèse modélisée par les auteurs par la possibilité de voir instantanément entrer de nouvelles compagnies d'assurance sur le marché, la situation étudiée par Rothschild et Stiglitz admet un équilibre, équilibre qui nous permet d'affirmer que, malgré la situation concurrentielle, les agents pâtissent de l'asymétrie d'information existant entre assuré et assureur. Mais le contrat étudié par Rothschild et Stiglitz (RS) n'est pas toujours un équilibre, et il est tout à fait possible que cette situation de concurrence de type Bertrand n'admette aucun équilibre en stratégies pures.

C'est ce dernier résultat qui a reçu toute l'attention des travaux postérieurs à RS. Deux sortes d'extensions ont suivi ce résultat. D'une part, reconnaître que l'inexistence d'un équilibre en stratégies pures était lié au caractère non efficace (au sens du second rang) de la solution concurrentielle (Crocker et Snow). Dasgupta et Maskin montrent qu'un tel jeu est susceptible d'admettre des équilibres en stratégies mixtes, la propriété de non efficacité pouvant s'appliquer, bien sûr, à leurs résultats. D'autre part, tout un autre courant de la littérature a tenté de modifier le concept d'équilibre utilisé par RS, des tentatives initiales de définition d'équilibres différents (Wilson, Miazaky, Riley) ont été suivies par des modélisations de processus concurrentiels sous forme de jeu séquentiel (Grossman, Hellwig). Un des apports de cette littérature est donc de montrer à quel point l'équilibre d'un jeu est sensible aux hypothèses faites sur la séquentialité des choix.

Dans cet article, on souhaite revenir sur l'un des problèmes posés par RS, à savoir l'existence et l'inefficacité d'un équilibre en stratégies pures. Le point de départ de cette réflexion est le fait que les résultats d'inefficacité obtenus par Rothschild et Stiglitz ne sont pas généraux. Il existe toute une classe de fonctions d'utilité qui vérifient des propriétés assez étonnantes: d'une part, le jeu étudié par Rothschild et Stiglitz admet toujours un équilibre en stratégies pures, et d'autre part le contrat d'équilibre est efficace au sens du premier rang. Comme on le verra, dans ce cas, la solution d'information asymétrique coïncide avec celle d'information parfaite. Ce résultat est vrai dès lors que le bien-être du principal, qui, dans notre problème, n'est pas informé, est indépendant du 
type de l'agent. Sous cette hypothèse, la solution d'information parfaite satisfait les contraintes d'incitation et l'asymétrie d'information ne joue aucun rôle pour peu que la concurrence entre principaux soit suffisamment vive.

Dire que l'utilité du principal ne dépend pas du type de l'agent revient à dire que le principal ne court aucun risque quand il propose la solution d'information parfaite puisque son profit est, quelque soit le type de l'agent, nul. Il en est de même si le contrat d'information parfaite du jeu étudié est tel que le profit du principal est positif ou nul pour les échanges offerts, qu'ils soient choisis par un agent d'un certain type ou un autre.

Cette remarque nous conduit à définir la "précision d'une structure d'information". Tout échange entre deux individus est en fait un échange de bien-être. Supposer qu'il existe une asymétrie d'information entre deux parties contractantes, un principal et un agent, revient donc à supposer que l'échange des bien-êtres est inconnu. De plus, ces parties ne peuvent s'accorder que sur des variables observables.

L'échange en asymétrie d'information est alors modélisé de la façon suivante. Imaginons que y soit la variable observable de l'échange. Pour un y donné, la situation présente une asymétrie d'information si au moins l'une des parties ne connaît pas exactement le bienêtre qu'elle obtient ou que l'autre partie obtient pour un signal y, alors même que cette dernière est informée de ces paramètres. On peut dès lors supposer que le bien-être du principal dépend d'une variable connue de l'agent seul, $\theta$ (dans ce type de modèle c'est donc l'agent qui est dispose d'une information privée) et s'écrit $V(y, \theta)$, et de la même façon le bien-être de l'agent dépend de son information privée, soit $\mathrm{U}(\mathrm{y}, \theta)$. Connaître (respectivement de pas connaître ) $\theta$ revient donc à connaître (respectivement ne pas connaître) exactement le bien-être lié à l'observation de y.

Imaginons maintenant que deux structures de signaux soit possibles. Par exemple, observer une classe de signaux $\alpha$ qui donne les bien-êtres $\mathrm{V}_{\alpha}(\mathrm{y}, \theta)$ et $\mathrm{U}_{\alpha}(\mathrm{y}, \theta)$ ou un classe de signaux $\alpha^{*}$ qui donne les bien-êtres $V_{\alpha^{*}}(y, \theta)$ et $U_{\alpha^{*}}(y, \theta)$. La définition proposée en section IV de cet article selon laquelle $\alpha^{*}$ est plus précise que $\alpha$ équivaut à dire que le profit du profit du principal $\mathrm{V}_{\alpha^{*}}(\mathrm{y}, \theta)$ varie moins en fonction de $\theta$ que $\mathrm{V}_{\alpha}(\mathrm{y}, \theta)$. Ceci revient intuitivement à supposer que le profit du principal est moins incertain. On montre alors que, plus la structure d'information est précise au sens du principal, plus le bien-être obtenu par l'agent à l'équilibre RS est important. Le cas étudié en section III apparaît donc bien comme le cas limite d'une étude de structure d'information. Par contre, on ne peut affirmer que l'équilibre RS existe plus souvent si la structure d'information est plus précise. Autrement dit, la solution de RS dans une structure d'information très précise au sens défini peut ne pas être un optimum de second rang, et dominer la solution RS dans une structure d'information moins précise, cette dernière étant optimale au sens du second rang. 
Quel est alors l'apport de ce travail ?

Il existe un lien entre structure d'information et efficacité du marché. Plus le principal est informé, non pas du bien-être que l'agent tire de la relation, mais plutôt du bien-être que lui-même tire de la relation, moins les contraintes d'incitation de l'agent sont actives, quand le principal souhaite se comporter au mieux des intérêts de l'agent, et donc plus l'équilibre RS est efficace. Le cas limite est étudié en première section, quand le profit du principal ne dépend pas du type de l'agent, l'équilibre de RS est efficace au sens du premier rang.

L'article est organisé de la façon suivante. En première section, le jeu de RS est présenté, ainsi que la définition d'un équilibre dans ce jeu. Puis (section II) on rappelle les résultats du modèle étudié par RS. En section III, on s'intéresse au cas où le bien-être du principal ne dépend pas du type de l'agent. L'existence d'un équilibre et l'efficacité de cet équilibre sont prouvées, sous des hypothèses peu restrictives. Enfin, dans la dernière section (IV), on propose une interprétation de ces résultats, en s'intéressant à des structures d'information différentes.

\section{Section I: Le jeu étudié, définition d'un équilibre}

On s'intéresse à un modèle principal-agent de pure information cachée qui peut être décrit de la façon suivante. Un échange observable est un élément y appartenant à un ensemble donné $Y$, convexe et compact. On appelle $\theta$ le type de l'agent, dont le principal ignore la valeur, et sur lequel on fait les hypothèses suivantes:

. dans le cas discret, $\theta \in\left\{\theta_{1}, \theta_{2}\right\}$ et l'on note $\gamma_{i}$ la probabilité que l'agent soit de type $\theta_{i}$, $i=1,2$, cette probabilité étant connue du principal.

. dans le cas continu, $\theta$ appartient à un intervalle $\left[\theta_{2}, \theta_{1}\right], f(\theta)$ est la densité de la variable $\theta$ sur cet intervalle et $F(\theta)$ sa loi de répartition.

Si l'échange est y, le bien-être obtenu par les deux parties est de la forme: le principal obtient $\mathrm{V}(\mathrm{y}, \theta)$ et l'agent obtient $\mathrm{U}(\mathrm{y}, \theta)$ si le type de l'agent est $\theta$. $U$ et $\mathrm{V}$ sont des fonctions continues de $\mathrm{Y}$. Dans le cas discret, on dira qu'un couple d'échanges $\left(\mathrm{y}_{1}, \mathrm{y}_{2}\right)$ satisfait IC si l'agent préfère y1 quand son type est $\theta_{1}$ et $y_{2}$ dans le cas inverse:

$\mathrm{U}\left(\mathrm{y}_{1}, \theta_{1}\right) \geq \mathrm{U}\left(\mathrm{y}_{2}, \theta_{1}\right)$ et $\mathrm{U}\left(\mathrm{y}_{2}, \theta_{2}\right) \geq \mathrm{U}\left(\mathrm{y}_{1}, \theta_{2}\right)$.

Un contrat dans ce modèle principal-agent simple est alors la donnée de plusieurs échanges, éléments de Y. Ce contrat est proposé par le principal, et l'agent connaissant son type, choisit l'un des échanges du contrat. Dans le cas discret où le type de l'agent ne peut prendre que deux valeurs distinctes, on supposera qu'un contrat est la donnée de 
deux échanges. Dans le cas continu, on notera contrat un ensemble $\left\{y(\theta), \theta \in\left[\theta_{2}, \theta_{1}\right]\right\}$. Enfin remarquons que l'agent avant de choisir un échange apprend la valeur de son type.

\section{Le jeu étudié:}

On s'intéresse au jeu suivant:

Le principal $A$ et le principal $B$ proposent simultanément un contrat $\left(C_{A}\right.$ contrat du principal $\mathrm{A}, \mathrm{C}_{\mathrm{B}}$ contrat du principal $\mathrm{B}$ ) à l'agent. L'agent choisit un échange dans l'ensemble total proposé, ou encore il choisit le contrat d'un principal, puis un échange dans le contrat offert par ce principal.

Définition d'un équilibre de Nash en stratégies pures du jeu (équilibre RS), dans le cas où $\theta \in\left\{\theta_{2}, \theta_{1}\right\}$

Notons $x^{i_{k h}}$ la probabilité que l'agent si son type est $\theta_{i}(i=1,2)$ choisisse le $k^{i e ̀ m e}$ élément $(k=1,2)$ du contrat offert par le principal $h, h=A, B$.

$\mathrm{C}_{\mathrm{A}}{ }^{*}=\left\{\mathrm{y}_{1 \mathrm{~A}}{ }^{*}, \mathrm{y}_{2 \mathrm{~A}}{ }^{*}\right\}, \mathrm{C}_{\mathrm{B}}{ }^{*}=\left\{\mathrm{y}_{1 \mathrm{~B}}{ }^{*}, \mathrm{y}_{2 \mathrm{~B}}{ }^{*}\right\}, \mathrm{x}^{\mathrm{i}^{*}}=\left(\mathrm{x}^{\mathrm{i}} 1 \mathrm{~A}^{*}, \mathrm{x}^{\mathrm{i}} 2 \mathrm{~A}^{*}, \mathrm{x}^{\mathrm{i}} 1 \mathrm{~B}^{*}, \mathrm{x}^{\mathrm{i}}{ }_{2 \mathrm{~B}}{ }^{*}\right), \mathrm{i}=1,2$ est un équilibre de Nash du jeu en stratégies pures si:

i) $x^{i^{*}}=x^{i}\left(C_{A}^{*}, C_{B}^{*}\right)$

avec

$x^{i}\left(C_{A}, C_{B}\right)=\left(x^{i}{ }_{1 A}\left(C_{A}, C_{B}\right), x^{i}{ }_{2 A}\left(C_{A}, C_{B}\right), x^{i}{ }_{1 B}\left(C_{A}, C_{B}\right), x^{i}{ }_{2 B}\left(C_{A}, C_{B}\right)\right)$ solution de:

$$
\begin{aligned}
& \operatorname{Max} x_{1 A}^{i} U\left(y_{1 A}, \theta_{i}\right)+x_{2 A}^{i} U\left(y_{2 A}, \theta_{i}\right)+x_{1 B}^{i} U\left(y_{1 B}, \theta_{i}\right)+x_{2 B}^{i} U\left(y_{2 B}, \theta_{i}\right) \\
& \left(x^{\mathrm{i}}{ }_{1 A}, x^{\mathrm{i}}{ }_{2 \mathrm{~A}}, \mathrm{x}^{\mathrm{i}}{ }_{1 \mathrm{~B}}, \mathrm{x}^{\mathrm{i}}{ }_{2 \mathrm{~B}}\right) \\
& \text { SC } \\
& x^{i_{j}} \in[0,1], j=1 A, 2 A, 1 B, 2 B \\
& x^{i_{1 A}}+x^{i_{2 A}}+x^{i_{1 B}}+x^{i_{2 B}}=1
\end{aligned}
$$

L'agent de type $\theta$ choisit l'échange qui lui donne le plus grand niveau de bien-être, quel que soit les échanges offerts.

ii) On a:

$$
\begin{aligned}
& \Pi_{A}\left(C_{A}{ }^{*}, C_{B}{ }^{*}\right) \geq \Pi_{A}\left(C_{A}, C_{B}{ }^{*}\right) \text { pour tout } C_{A} \\
& \Pi_{B}\left(C_{A}{ }^{*}, C_{B}{ }^{*}\right) \geq \Pi_{B}\left(C_{A}{ }^{*}, C_{B}\right) \text { pour tout } C_{B}
\end{aligned}
$$

avec pour $\mathrm{i}=1,2$ et $\mathrm{K}=\mathrm{A}, \mathrm{B}$ :

$$
\begin{aligned}
& v\left(C_{K}, \theta_{i}\right)=x_{1 K}^{i_{1 K}}\left(C_{A}, C_{B}\right) V\left(y_{1 K}, \theta_{i}\right)+x^{i_{2 K}}\left(C_{A}, C_{B}\right) V\left(y_{2 K}, \theta_{i}\right) \\
& \Pi_{K}\left(C_{A}, C_{B}\right)=\gamma_{1} v\left(C_{K}, \theta_{1}\right)+\gamma_{2} v\left(C_{K}, \theta_{2}\right)
\end{aligned}
$$


Connaissant la règle de décision de l'agent et le contrat offert par son concurrent $B, l e$ principal A maximise son espérance de bien-être. Le principal B fait de même.

Section II: Propriété et existence d'un équilibre en stratégies pures, un rappel des résultats de $\mathrm{RS}$

L'apport central de RS, bien que ces derniers n'utilisent pas la notion d'équilibre de Nash, est de montrer que, si le jeu étudié admet un équilibre en stratégies pures, alors les deux principaux offrent le contrat $\left\{\mathrm{y}_{1} \mathrm{RS}_{,} \mathrm{y}_{2} \mathrm{RS}\right\}$, solution du programme suivant que l'on notera $P(\lambda)$, avec $\lambda \in] 0,1[$ :

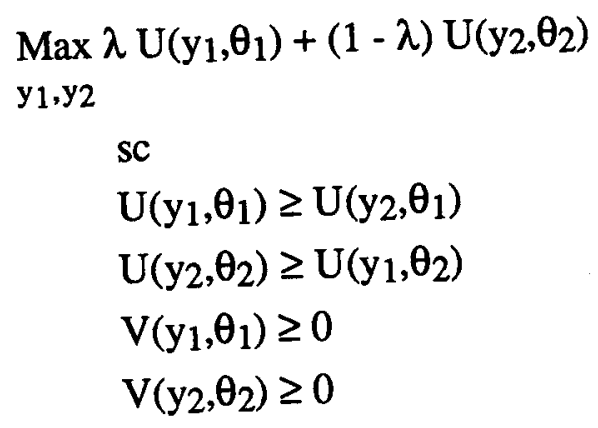

Le contrat d'équilibre du jeu RS rend maximum le bien-être de l'agent quel que soit son type sachant qu'aucun échange ne réalise des pertes à l'équilibre. Autrement dit, si l'agent quand son type est $\theta_{1}$ préfère $y_{1}$ à y (première contrainte d'incitation) le bien-être du principal est au moins nul $\left(\mathrm{V}\left(\mathrm{y}_{1}, \theta_{1}\right) \geq 0\right)$. De même pour l'agent si son type est $\theta_{2}$.

Quand le programme ci dessus admet plusieurs solutions, on retiendra celle qui donne le profit total le plus grand pour les principaux:

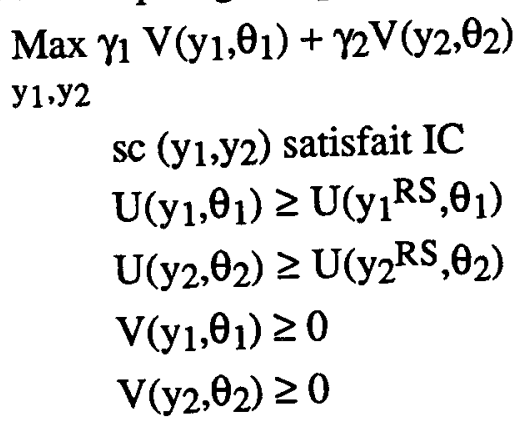

\section{Lemme 1:}

La solution du programme ci-dessus est unique en terme de bien-être pour l'agent. Elle est indépendante des valeurs prises par le paramètres $\lambda$, dès lors que celui-ci est 
strictement compris entre 0 et 1 . Si $\lambda=1$, alors $\left(\mathrm{y}_{1} \mathrm{RS}, \mathrm{y}_{2} \mathrm{RS}\right)$ est une des solutions de $P(1)$, de même si $\lambda=0$.

\section{Preuve du lemme 1:}

Notons $y_{i} R S$ la solution du programme $P_{i}$ :

$\operatorname{Max} U\left(y_{i}, \theta_{i}\right)$

$\mathrm{y}_{1}, \mathrm{y}_{2}$

$$
\begin{aligned}
& s c \\
& U\left(y_{1}, \theta_{1}\right) \geq U\left(y_{2}, \theta_{1}\right) \\
& U\left(y_{2}, \theta_{2}\right) \geq U\left(y_{1}, \theta_{2}\right) \\
& V\left(y_{1}, \theta_{1}\right) \geq 0 \\
& V\left(y_{2}, \theta_{2}\right) \geq 0
\end{aligned}
$$

Notons $y_{i}(\lambda), i=1,2$, les échanges solution du programme $P(\lambda)$.

Pour une valeur de $\left.\lambda_{i} \in\right] 0,1\left[\right.$ donné, $U\left(y_{i}{ }^{R S}, \theta_{1}\right)<U\left(y_{i}(\lambda), \theta_{i}\right)$ est impossible puisque le contrat $\left\{y_{i}(\lambda), i=1,2\right\}$ satisfait toutes les contraintes du programme $P_{i}$.

On a donc $\mathrm{U}\left(\mathrm{y}_{\mathrm{i}} \mathrm{RS}^{\mathrm{B}}, \theta_{1}\right) \geq \mathrm{U}\left(\mathrm{y}_{\mathrm{i}}(\lambda), \theta_{\mathrm{i}}\right)$

Montrons de plus que $\left\{y_{1}{ }^{R S}, y_{2}{ }^{R S}\right\}$ satisfait les contraintes du programme $P(\lambda)$ :

Les deux dernières contraintes sont bien sûr satisfaites, $V\left(y_{i}{ }^{R S}, \theta_{i}\right) \geq 0$.

Imaginons que l'une des contraintes d'incitation soit violée $\operatorname{par}\left(\mathrm{y}_{1} \mathrm{RS}_{, \mathrm{y}_{2}} \mathrm{RS}\right)$ :

$\mathrm{U}\left(\mathrm{y}_{1}{ }^{\mathrm{RS}}, \theta_{2}\right)>\mathrm{U}\left(\mathrm{y}_{2}{ }^{\mathrm{RS}}, \theta_{2}\right)$

Il existe alors $\mathrm{y}_{2},\left(\mathrm{y}_{1}{ }^{\mathrm{RS}}, \mathrm{y}_{2}\right)$ solution de $\mathrm{P}_{1}$ qui vérifie:

$\mathrm{U}\left(\mathrm{y}_{2}, \theta_{2}\right) \geq \mathrm{U}\left(\mathrm{y}_{1}{ }^{\mathrm{RS}}, \theta_{2}\right)>\mathrm{U}\left(\mathrm{y}_{2}{ }^{\mathrm{RS}}, \theta_{2}\right)$

$\mathrm{U}\left(\mathrm{y}_{1}{ }^{\mathrm{RS}}, \theta_{1}\right) \geq \mathrm{U}\left(\mathrm{y}_{2}, \theta_{1}\right)$

Mais alors $\left(y_{1}{ }^{R S}, y_{2}\right\}$ donne à l'agent $\theta_{2}$ un bien-être strictement plus grand que $\left\{y_{1} R S\right.$, $\left.y_{2}{ }^{R S}\right\}$ ce qui est impossible. On en déduit que $\left\{y_{i} R S, i=1,2\right\}$ est solution du programme $P(\lambda)$ pour tout $\lambda$.

Remarquons que dans le modèle étudié par RS l'une des contraintes d'incitation du programme ci-dessus est active. Dans ce cas, le contrat d'équilibre est inefficace au sens du premier rang: l'un des agents au moins souffre de l'asymétrie d'information, et son bien-être est inférieur à celui qu'il obtiendrait dans un jeu identique, mais dans lequel l'information serait parfaite.

Mais le contrat d'équilibre RS n'est pas toujours un équilibre du jeu. En effet, comme les contraintes d'incitations sont actives, il est possible de donner au moins à l'un des types d'agent une utilité plus élevée en réalisant des gains. Il existe donc un échange y et un type d'agent, $\theta_{\mathrm{i}}$ tel que:

$$
\mathrm{V}\left(\mathrm{y}, \theta_{\mathrm{i}}\right)>0 \text { et } \mathrm{U}\left(\mathrm{y}, \theta_{\mathrm{i}}\right)>\mathrm{U}\left(\mathrm{y}_{\mathrm{i}}^{\mathrm{RS}}, \theta_{\mathrm{i}}\right)
$$


Bien sûr, par définition de la solution de RS, si l'un des principaux offre de façon isolée y quand l'autre principal joue le contrat d'équilibre, les agents $\theta_{j}$ préfèreront y et induiront par ce choix un profit $\mathrm{V}\left(\mathrm{y}, \theta_{\mathrm{j}}\right)<0$. Mais il existe peut-être un contrat $\left\{\mathrm{y}_{1}, \mathrm{y}_{2}\right\}$ qui donne un bien-être plus grand à l'agent quel que soit son type, réalise des gains sur l'une des catégorie d'agents et des pertes sur l'autre catégorie, et qui engendre une espérance de bien-être strictement positive pour le principal. Si un tel contrat existe, alors n'importe quelle compagnie d'assurance proposera ce contrat si les compagnies concurrentes offrent le contrat RS.

Autrement dit, le contrat RS n'est pas un équilibre s'il existe un contrat $\left\{\mathrm{y}_{1}, \mathrm{y}_{2}\right\}$ satisfaisant IC qui vérifie :

$$
\begin{aligned}
& \mathrm{U}\left(\mathrm{y}_{1}, \theta_{1}\right)>\mathrm{U}\left(\mathrm{y}_{1} \mathrm{RS}_{,}, \theta_{1}\right) \\
& \mathrm{U}\left(\mathrm{y}_{2}, \theta_{2}\right)>\mathrm{U}\left(\mathrm{y}_{2}{ }^{\mathrm{RS}}, \theta_{2}\right) \\
& \gamma_{1} \mathrm{~V}\left(\mathrm{y}_{1}, \theta_{1}\right)+\gamma_{2} \mathrm{~V}\left(\mathrm{y}_{2}, \theta_{2}\right)>\gamma_{1} \mathrm{~V}\left(\mathrm{y}_{1}{ }^{\mathrm{RS}}, \theta_{1}\right)+\gamma_{2} \mathrm{~V}\left(\mathrm{y}_{2}{ }^{\mathrm{RS}}, \theta_{2}\right)
\end{aligned}
$$

Sachant que l'agent si son type est $\theta_{i}$ préfère l'échange $y_{i}$ du contrat, $i=1,2$, ce contrat donne à l'agent quel que soit son type un bien-être plus élevé que la proposition RS et engendre pour le principal qui le propose une espérance de bien-être non négative. Remarquons que si un tel contrat existe, il fait des pertes sur une catégorie d'agent et des gains sur l'autre catégorie d'agent.

Clairement, quand le jeu étudié n'admet pas d'équilibre, le contrat RS n'est pas efficace au sens du second rang. L'une des catégories d'agent a intérêt à financer les pertes faites sur l'autre catégorie. Dans ce cas la concurrence ne peut, parce quelle refuse de faire des pertes sur l'une des catégories d'agent, implémenter un échange efficace de second rang. Le résultat est important, car il montre que concurrence et efficacité ne vont pas toujours de pair quand le marché est caractérisé par des problèmes d'asymétrie d'information. Il est dès lors souhaitable d'encourager les compagnies d'assurance à se regrouper et d'imposer au monopole ainsi constitué de ne faire aucun profit. On comprend bien que modifier la notion d'équilibre du jeu ne change en rien l'essence de ce résultat.

Le but du travail ci-dessous est de préciser ces résultats dans un cadre plus général que celui étudié par RS, et en particulier d'établir sous quelles conditions ces résultats sont vrais.

Montrons que le contrat RS est un équilibre du jeu dès lors que la propriété (P) est vraie Propriété (P): Il n'existe aucun contrat $\left(\mathrm{y}_{1}, \mathrm{y}_{2}\right) \in \mathrm{Y}^{2}$ et satisfaisant IC tel que.

$$
\begin{aligned}
& \mathrm{U}\left(\mathrm{y}_{1}, \theta_{1}\right) \geq \mathrm{U}\left(\mathrm{y}_{1}{ }^{\mathrm{RS}}, \theta_{1}\right) \\
& \mathrm{U}\left(\mathrm{y}_{2}, \theta_{2}\right) \geq \mathrm{U}\left(\mathrm{y}_{2}{ }^{\mathrm{RS}}, \theta_{2}\right) \\
& \gamma_{1} \mathrm{~V}\left(\mathrm{y}_{1}, \theta_{1}\right)+\gamma_{2} \mathrm{~V}\left(\mathrm{y}_{2}, \theta_{2}\right)>0
\end{aligned}
$$


Hypothèse $\mathrm{H}_{1}$ :

Pour tout contrat $\left(\mathrm{y}_{1}, \mathrm{y}_{2}\right) \in \mathrm{Y}^{2}$ et satisfaisant IC, pour tout $\varepsilon>0$ petit, il existe $\left(\mathrm{y}_{1}^{\prime}, \mathrm{y}_{2}^{\prime}\right)$ $\in \mathrm{Y}^{2}$ satisfaisant IC tel que:

$\mathrm{U}\left(\mathrm{y}^{\prime}{ }_{\mathrm{i}}, \theta_{\mathrm{i}}\right)>\mathrm{U}\left(\mathrm{y}_{\mathbf{i}}, \theta_{\mathrm{i}}\right)$

$\mathrm{V}\left(\mathrm{y}_{\mathbf{i}}^{\prime}, \theta_{\mathrm{i}}\right) \geq \mathrm{V}\left(\mathrm{y}_{\mathbf{i}}, \theta_{\mathrm{i}}\right)-\varepsilon$

\section{Proposition 1:}

Si $\mathrm{H}_{1}$ est vérifiée, $\left(\mathrm{y}_{1} \mathrm{RS}, \mathrm{y}_{2}{ }^{\mathrm{RS}}\right)$ est une allocation d'équilibre du jeu si et seulement si $\mathrm{P}$ est vraie.

\section{Preuve de la proposition: voir annexe 1}

$\mathrm{H}_{2}$ :

Pour tout $\varepsilon>0$, petit et tout échange $y \in Y$, et pour tout $i$, il existe un échange $y^{\prime} \in Y$ telle que:

$\mathrm{U}\left(\mathrm{y}^{\prime}, \theta_{\mathrm{i}}\right)>\mathrm{U}\left(\mathrm{y}, \theta_{\mathrm{i}}\right)$

$\mathrm{U}\left(\mathrm{y}, \theta_{-\mathrm{i}}\right)>\mathrm{U}\left(\mathrm{y}^{\prime}, \theta_{-\mathrm{i}}\right)$

$\mathrm{V}\left(\mathrm{y}^{\prime}, \theta_{\mathrm{i}}\right) \geq \mathrm{V}\left(\mathrm{y}, \theta_{\mathrm{i}}\right)-\varepsilon$

\section{Proposition 2:}

$\mathrm{Si}_{2}$ est vraie, aucun échange ne réalise des gains ( strictement positifs) à l'équilibre, si elle est choisie, et l'équilibre RS domine au sens de Pareto l'ensemble des équilibres.

Cette hypothèse nous dit que pour tout échange $y$, on peut trouver un échange $y$ ' telle que l'agent i préfère l'échange y' à l'échange y, contrairement à l'autre type d'agent, et que cet échange ne donne pas au principal un bien-être très différent de celui qu'il obtient si le type de l'agent est $\theta_{i}$ et que le choix est $y$.

Dès lors que $y^{\prime}$ est proche de $y$ et que les fonctions $U$ et $V$ sont continues, cette hypothèse reflète la propriété selon laquelle les surfaces d'indifférence des agents ne sont jamais tangentes. En effet dans ce cas, on peut toujours trouver un échange y' à proximité d'un point y qui vérifie les deux premières conditions de l'hypothèse $S C$, et $V\left(y^{\prime}, \theta_{i}\right)$ est proche de $\mathrm{V}\left(\mathrm{y}, \theta_{\mathrm{i}}\right)$ (plus grand, plus petit, ou plus faible).

\section{Preuve de la proposition 2:}

Cette hypothèse a deux conséquences très fortes:

Conséquence 1: aucun échange ne réalise des gains à l'équilibre ( si il est choisi). 
Pour montrer ceci, notons $C_{A}$ et $C_{B}$ les contrats proposés à l'équilibre par les deux principaux. Supposons que l'échange $\mathrm{y}_{1 \mathrm{~A}}$ réalise des gains et est choisi:

. $\mathrm{V}\left(\mathrm{y}_{1 \mathrm{~A}}, \theta_{1}\right)>0$

. $\mathrm{x}_{\mathrm{A}}\left(\theta_{1}\right)>0$.

On a donc $\mathrm{U}\left(\mathrm{y}_{1 \mathrm{~A}}, \theta_{1}\right) \geq \mathrm{U}\left(\mathrm{y}_{1 \mathrm{~B}}, \theta_{1}\right)$.

On sait qu'il existe un échange $y(\varepsilon)$ qui satisfait:

$\mathrm{U}\left(\mathrm{y}, \theta_{1}\right)>\mathrm{U}\left(\mathrm{y}_{1 \mathrm{~A}}, \theta_{1}\right)$

$\mathrm{U}\left(\mathrm{y}_{1 \mathrm{~A}}, \theta_{2}\right)>\mathrm{U}\left(\mathrm{y}, \theta_{2}\right)$

$\mathrm{V}\left(\mathrm{y}, \theta_{1}\right) \geq \mathrm{V}\left(\mathrm{y}_{1 \mathrm{~A}}, \theta_{1}\right)-\varepsilon$.

avec $\varepsilon>0$

Remarquons que le contrat $\left\{\mathrm{y}(\varepsilon), \mathrm{y}_{2 \mathrm{~A}}\right\}$ satisfait les contraintes d'incitations:

$\mathrm{U}\left(\mathrm{y}(\varepsilon), \theta_{1}\right)>\mathrm{U}\left(\mathrm{y}_{2 \mathrm{~A}}, \theta_{1}\right)$

$\mathrm{U}\left(\mathrm{y}_{2 \mathrm{~A}}, \theta_{2}\right) \geq \mathrm{U}\left(\mathrm{y}_{1 \mathrm{~A}}, \theta_{2}\right) \geq \mathrm{U}\left(\mathrm{y}(\varepsilon), \theta_{2}\right)$

Dès lors que $\mathrm{x}_{\mathrm{A}}\left(\theta_{1}\right)<1$, il existe $\varepsilon$ tel que proposer $\left\{\mathrm{y}(\varepsilon), \mathrm{y}_{2 \mathrm{~A}}\right\}$ assure au principal $\mathrm{A}$ un profit strictement plus grand que son profit d'équilibre.

Si $x_{A}\left(\theta_{1}\right)=1$, la même remarque s'applique aussi au principal $B$ ( $\operatorname{proposer}\left\{y(\varepsilon), y_{2} B\right.$ ), puisqu'on est sûr qu'à l'équilibre $\mathrm{x}_{\mathrm{B}}\left(\theta_{1}\right)=0$.

\section{Conséquence 2:}

Aucun échange ne réalise des pertes à l'équilibre si il est choisi.

Cette conséquence découle directement de la première: en effet, si aucun échange choisi ne réalise des gains et s'il existe un échange choisi qui réalise des pertes, alors l'un des principaux obtient une espérance de bien-être négative à l'équilibre, ce qui est impossible, il préfèrera offrir le contrat $\mathrm{RS}$, qui lui assure une espérance d'utilité nulle.

Ces deux conséquences de l'hypothèse RS nous permettent d'affirmer que l'équilibre RS domine au sens de Pareto l'ensemble des équilibres.

QED

\section{Le lien entre $P$ et la notion d'optimum de second rang.}

Si $P$ est vraie alors $V\left(y_{i}^{R S}, \theta_{i}\right)=0, i=1,2$, il n'existe aucun échange qui donne à l'agent quel que soit son type un bien-être plus grand que le contrat RS, susceptible de réaliser une espérance de profit strictement positive pour le principal. L'hypothèse $\mathrm{H}_{1}$ nous permet d'affirmer que tout contrat satisfaisant IC tel que l'agent quel que soit son type obtient un bien-être strictement plus grand que celui que lui donne le contrat RS engendre une espérance de profit strictement négative. Mais, par contre, il peut exister un contrat 
satisfaisant IC, qui engendre une espérance de profit nulle et donne à un type d'agent un bien-être supérieur à son niveau d'utilité pour le contrat RS, alors que l'autre type d'agent obtient exactement le niveau de bien-être du contrat RS. On est alors sûr que l'agent qui obtient un bien-être strictement plus grand engendre des pertes sur l'échange qu'il choisira, l'échange de l'autre type d'agent réalisant de ce fait des gains. Ceci apparaît pourtant comme un cas pathologique.

Si un tel contrat n'existe pas, alors RS est un équilibre si et seulement si il est un optimum de second rang.

\section{Section III: Le cas de valeurs privées}

On s'intéresse dans cette partie à l'existence de l'équilibre RS dans un cas particulier, qui a reçu le nom de valeurs privées. Cette terminologie est en général utilisée en théorie des enchères et désigne le fait que les offreurs valuent le bien qu'ils cherchent à acquérir de façon indépendante. Ici nous l'employons pour désigner simplement le fait que le profit que le principal tire de la relation est indépendant de l'information privée de l'agent, à savoir:

Pour tout $y \in Y$, le profit du principal est $V(y)$ et l'utilité de l'agent est $U(y, \theta)$ si son type est $\theta$.

Ce cas particulier présente d'étonnantes propriétés. Sous des hypothèses assez peu restrictives et dans le cas où le type de l'agent appartient à l'ensemble $\left\{\theta_{2}, \theta_{1}\right\}$ l'équilibre RS existe, est unique et efficace au sens du premier rang. L'asymétrie d'information ne joue aucun rôle sur le contrat offert à l'équilibre du jeu étudié.

\section{Définition:}

Soit $\mathrm{y}^{*}(\theta)$ l'échange solution du programme:

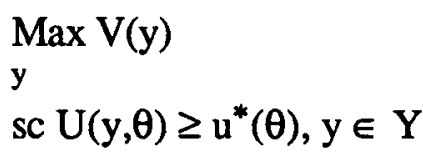

avec $\mathrm{u}^{*}(\theta)$ fonction valeur du programme:

$$
\begin{aligned}
& \operatorname{Max} U(y, \theta) \\
& y \\
& \operatorname{sc} V(y) \geq 0, y \in Y
\end{aligned}
$$

$\mathrm{y}^{*}(\theta)$ est l'échange qui d'une part, rend maximum le bien-être de l'agent (qui sera noté par la suite $\mathrm{u}^{*}(\theta)$ ) sachant que le principal obtient au moins un bien-être nul, et d'autre part donne au principal le plus grand bien-être dans l'ensemble des contrats qui donnent à l'agent $\mathrm{u}^{*}(\theta)$.

Si chaque principal connaissait le type de l'agent, l'intensité du processus concurrentiel étudié dans ce jeu garantit que chaque principal offre à l'équilibre le contrat $\mathrm{y}^{*}(\theta)$ à l'agent 
quand son type est $\theta$. Le but de cette section est de montrer qu'à l'équilibre du jeu d'information asymétrique, chaque principal offre $\mathrm{y}^{*}(\theta)$ à l'agent si son type est $\theta$.

\section{Hypothèses}

$\mathrm{H}_{3}: \mathrm{y}^{*}(\theta)$ existe pour tous les $\theta$ étudiés.

$\mathrm{H}_{4}$ : $\mathrm{U}$ et $\mathrm{V}$ sont deux fonctions concaves de $\mathrm{y}$

$\mathrm{H}_{5}$ : Il existe au moins un $\mathrm{y} \in \mathrm{Y}$ tel que $\mathrm{V}(\mathrm{y})>\operatorname{Max}\left(\mathrm{v}^{*}\left(\theta_{1}\right), \mathrm{v}^{*}\left(\theta_{2}\right)\right)$

Les deux hypothèses $\mathrm{H}_{3}$ et $\mathrm{H}_{5}$ posent que le problème n'est pas trivial. $\mathrm{H}_{4}$ est une hypothèse standard dans la littérature principal-agent, utile en général pour obtenir l'unicité d'un équilibre.

\section{Proposition 3:}

Sous $\mathrm{H}_{3}$, le jeu étudié admet au moins un équilibre. Cet équilibre est efficace au sens du premier rang. Le principal propose à l'agent de type $\theta_{\mathrm{i}}$ l'échange qu'il lui offrirait dans le même jeu s'il était sûr que son type est $\theta_{\mathrm{i}}$ :

$C^{*}=\left\{y^{*}\left(\theta_{i}\right), i=1,2\right\}$ et l'agent choisit le principal $K$ avec une probabilité $\mathrm{xK}^{*}\left(\theta_{i}\right)$, telles que $\mathrm{x}_{\mathrm{A}}{ }^{*}\left(\theta_{\mathrm{i}}\right)+\mathrm{x}_{\mathrm{B}}{ }^{*}\left(\theta_{\mathrm{i}}\right)=1, \mathrm{x}_{\mathrm{A}}{ }^{*}\left(\theta_{\mathrm{i}}\right) \geq 0, \mathrm{x}_{\mathrm{B}}{ }^{*}\left(\theta_{\mathrm{i}}\right) \geq 0, \mathrm{i}=1,2$

Le jeu RS sous nos hypothèses admet au moins un équilibre.Cet équilibre est efficace dans le sens suivant: il n'existe aucun échange qui augmente le bien-être d'un agent et donne une utilité positive au principal. L'équilibre du jeu RS en asymétrie d'information est un contrat qui rassemble l'échange du même jeu en information parfaite quand le type de l'agent est $\theta_{1}$ et celui du même jeu en information parfaite quand le type de l'agent est $\theta_{2}$. Aucun agent ne souffre de l'asymétrie d'information. On a donc sous l'hypothèse de valeurs privées, à la fois existence et efficacité du contrat d'équilibre RS.

\section{Preuve de la proposition 3:}

\section{Notations}

On note $u(C, \theta)$ l'utilité qu'obtient l'agent si son type est $\theta$ quand il a le choix entre deux échanges d'un contrat $C=\left(\mathrm{y}_{1}, \mathrm{y}_{2}\right)$. On peut aisément définir $\mathrm{u}(\mathrm{C}, \theta)$ :

$$
\mathrm{u}(\mathrm{C}, \theta)=\operatorname{Max}\left(\mathrm{U}\left(\mathrm{y}_{1}, \theta\right), \mathrm{U}\left(\mathrm{y}_{2}, \theta\right)\right)
$$

De la même façon, on note $\mathrm{v}(\mathrm{C}, \theta)$ le profit qu'obtiendrait le principal qui propose le contrat $C$ quand l'agent de type $\theta$ choisit ce contrat. On a:

$$
\text { si } U\left(y_{i}, \theta\right) \geq U\left(y_{j}, \theta\right), v(C, \theta)=V\left(y_{i}\right), i=1,2, j=1,2
$$


Si l'agent de type $\theta$ est indifférent entre les deux échanges du contrat offert par l'un des principaux, on suppose, comme il est usuel de le faire, que l'agent choisit l'échange que préfêre le principal.

Enfin on note $\mathrm{x}_{\mathrm{K}}(\theta)$ la probabilité pour que l'agent de type $\theta$ préfère un échange du contrat $\mathrm{C}_{\mathrm{K}}$ proposé par le principal $\mathrm{K}, \mathrm{K}=\mathrm{A}, \mathrm{B}$, à un échange du contrat offert par le principal concurrent. On a bien-sûr $x_{A}(\theta)+x_{B}(\theta)=1, x_{A}(\theta) \in[0,1]$.

Supposons que l'un des principaux (B) offre le contrat $\left\{y^{*}\left(\theta_{i}\right), i=1,2\right\}$ et montrons qu'il existe une règle de décision de l'agent, séquentiellement rationnelle, qui garantit que la meilleure réponse du principal concurrent (A) est d'offrir le même contrat.

Le profit d'équilibre du principal A s'écrit:

$$
\begin{aligned}
& \Pi_{A}\left(C^{*}, C^{*}\right)=\gamma_{1} x_{A}{ }^{*}\left(\theta_{1}\right) v^{*}\left(\theta_{1}\right)+\gamma_{2} x_{A}{ }^{*}\left(\theta_{2}\right) v^{*}\left(\theta_{2}\right) \\
& \operatorname{avec} x_{A}{ }^{*}\left(\theta_{i}\right) \in[0,1], i=1,2
\end{aligned}
$$

Un tel contrat est révélateur. En effet, on a par définition:

$$
\mathrm{U}\left(\mathrm{y}^{*}\left(\theta_{\mathrm{i}}\right), \theta_{\mathrm{i}}\right) \geq \mathrm{U}\left(\mathrm{y}^{*}\left(\theta_{\mathrm{j}}\right), \theta_{\mathrm{i}}\right) \text {, pour } \mathrm{i}=1,2 ; \mathrm{j}=1,2 \text {. }
$$

Considérons l'ensemble de tous les contrats $\mathrm{C}$ possibles et montrons que

$$
\Pi_{A}\left(C, C^{*}\right) \leq \Pi_{A}\left(C^{*}, C^{*}\right) \text {. }
$$

On sait que:

$$
\Pi_{A}\left(C, C^{*}\right)=\gamma_{1} x_{A}\left(\theta_{1}\right) v\left(C, \theta_{1}\right)+\gamma_{2} x_{A}\left(\theta_{2}\right) v\left(C, \theta_{2}\right)
$$

avec pour $\mathrm{i}=1,2$ :

$$
\begin{aligned}
& \text {. si } u\left(C, \theta_{i}\right)>u\left(C^{*}, \theta_{i}\right), x_{A}\left(\theta_{i}\right)=1 \\
& \text {. si } u\left(C, \theta_{i}\right)=u\left(C^{*}, \theta_{i}\right) \text {, on choisit } x_{A}\left(\theta_{i}\right)=x_{A}{ }^{*}\left(\theta_{i}\right) \\
& \text {. si } u\left(C, \theta_{i}\right)<u\left(C^{*}, \theta_{i}\right), x_{A}\left(\theta_{i}\right)=0
\end{aligned}
$$

Montrons que, quel que soit $i=1,2, x_{A}\left(\theta_{i}\right) v\left(C, \theta_{i}\right) \leq x_{A}{ }^{*}\left(\theta_{i}\right) v\left(C^{*}, \theta_{i}\right)$.

En effet, :

. si $u\left(C, \theta_{i}\right)>u\left(C^{*}, \theta_{i}\right), x_{A}\left(\theta_{i}\right)=1$, mais $v\left(C, \theta_{i}\right)<0$, donc $x_{A}\left(\theta_{i}\right) v\left(C, \theta_{i}\right)<$ $\mathrm{x}_{\mathrm{A}}{ }^{*}\left(\theta_{\mathrm{i}}\right) \mathrm{v}\left(\mathrm{C}^{*}, \theta_{\mathrm{i}}\right)$

. si $u\left(C, \theta_{i}\right)=u\left(C^{*}, \theta_{i}\right), x_{A}\left(\theta_{i}\right)=x_{A}{ }^{*}\left(\theta_{i}\right)$ mais par définition $v\left(C, \theta_{i}\right) \leq v\left(C^{*}, \theta_{i}\right)$ donc $\mathrm{x}_{\mathrm{A}}\left(\theta_{\mathrm{i}}\right) \mathrm{v}\left(\mathrm{C}, \theta_{\mathrm{i}}\right) \leq \mathrm{x}_{\mathrm{A}}{ }^{*} \mathrm{v}\left(\mathrm{C}^{*}, \theta_{\mathrm{i}}\right)$

. si $u\left(C, \theta_{i}\right)<u\left(C^{*}, \theta_{i}\right), x_{A}\left(\theta_{i}\right)=0, x_{A}\left(\theta_{i}\right) v\left(C, \theta_{i}\right)=0$ et donc $x_{A}\left(\theta_{i}\right) v\left(C, \theta_{i}\right) \leq$ $\mathrm{x}_{\mathrm{A}}^{*}\left(\theta_{\mathrm{i}}\right) \mathbf{v}\left(\mathrm{C}^{*}, \theta_{\mathrm{i}}\right)$

Le point clé de la proposition 3 réside dans le fait que le contrat $\left(y^{*}\left(\theta_{i}\right), i=1,2\right\}$ satisfait les contraintes d'incitation de l'agent, autrement dit que l'agent d'un type donné préfère son échange de premier rang à l'échange de l'autre. La concurrence fait donc disparaître le problème de sélection adverse qui existerait si le principal proposait un contrat à l'agent en cherchant à obtenir un bien-être le plus grand possible de la relation. Mais cette intuition selon laquelle la concurrence adoucit les problèmes d'incitations de l'agent, 
simplement car elle conduit à rendre maximum le bien-être de l'agent lui-même, reste vraie même lorsque l'utilité du principal dépend du type de l'agent. Dans ce cas un autre problème apparaît, et l'intuition est particulièrement claire sous l'hypothèse d'utilité transférable au moyen par exemple d'un paiement monétaire. Le paiement dans le cas concurrentiel qu'accorde le principal à un agent d'un type donné dépend justement de son type ( car il annule son bien-être), ce qui n'est pas vrai sous notre hypothèse de valeurs privées. C'est alors l'opportunité d'un paiement plus grand qui peut pousser l'agent à mentir quand on lui offre l'ensemble des échanges de premier rang. Autrement dit, c'est bien parce que l'utilité du principal dépend de façon donnée du type de l'agent que l'ensemble des échanges de premier rang ne satisfait plus les contraintes d'incitations de l'agent.

La proposition 4 nous permet d'affirmer que l'équilibre du jeu est unique, la proposition 5 montre que l'analyse peut être généralisée au cas où le type de l'agent peut prendre un nombre plus important de valeurs.

\section{Proposition 4:}

Sous $\mathrm{H}_{1}, \mathrm{H}_{3}, \mathrm{H}_{4}$ et $\mathrm{H}_{5}$, les paiements d'équilibre du jeu sont uniques: si $y_{i}$ est une échange proposé à l'équilibre et choisi par l'agent si son type est $\theta_{i}$ avec une probabilité non nulle, alors $\mathrm{U}\left(\mathrm{y}_{\mathrm{i}}, \theta_{\mathrm{i}}\right)=\mathrm{u}^{*}\left(\theta_{\mathrm{i}}\right)$ et $\mathrm{V}\left(\mathrm{y}_{\mathrm{i}}\right)=\mathrm{v}^{*}\left(\theta_{\mathrm{i}}\right)$.

Aucun des équilibres du jeu étudié n'est donc inefficace au sens du premier rang: les paiements d'équilibre du jeu sont uniques.

La preuve de la proposition 4 est reportée en annexe 2.

\section{Proposition 5:}

Sous $\mathrm{H}_{3}$, si chaque principal pense que le type de l'agent peut appartenir à l'intervalle $\left[\theta_{2}, \theta_{1}\right]$, et si les croyances de chaque principal sont représentées par une fonction de densité $f(\theta)$ sur cet intervalle, alors le jeu étudié admet au moins un équilibre. Les deux principaux offrent le contrat $C^{*}=\left\{\left(y^{*}(\theta), \theta \in \Theta\right\}\right.$ et l'agent si son type est $\theta$ choisit le contrat du principal $K$ avec une probabilité $\mathrm{xK}^{*}(\theta), \mathrm{K}=\mathrm{A}, \mathrm{B}$.

Le résultat de la proposition 3 se généralise au cas continu, dans lequel le type de l'agent peut prendre toutes les valeurs d'un intervalle.

\section{Preuve de la proposition 5:}

La preuve de la proposition 5 est la même que celle de la proposition 3. 
A l'équilibre, le profit de chaque principal s'écrit, s'ils offrent le contrat $\left\{\left(y^{*}(\theta), \theta \in \Theta\right\}\right.$

$$
\Pi_{A}\left(C^{*}, C^{*}\right)=\int_{\theta_{2}}^{\theta_{1}} v^{*}(u) h(u) d u
$$

où $h(\theta)$ est déduite des croyances initiales et de la stratégie d'équilibre de l'agent:

$$
h(\theta)=x_{A}^{*}(\theta) f(\theta)
$$

Soit $\mathrm{X}$ l'ensemble de tous les contrats possibles. Quand le principal A offre un contrat $\mathrm{C}$ alors que le principal concurrent propose $\mathrm{C}^{*}$, son bien-être prend les valeurs:

$$
\Pi_{A}\left(C, C^{*}\right)=\int_{\theta_{2}}^{\theta_{1}} v(C, u) l(u) d u
$$

Il existe alors une règle de décision de l'agent $\left(\mathrm{x}_{\mathrm{A}}(\theta) \in\left\{0, \mathrm{x}_{\mathrm{A}}{ }^{*}(\theta), 1\right\}\right)$ telle que:

$$
\begin{aligned}
& \text {.si } u\left(C, \theta_{i}\right)>u(C, \theta) \text {, alors } 1(\theta)=f(\theta) \text { et } v(C, \theta)<0 \\
& \text {.si } u(C, \theta)=u\left(C^{*}, \theta\right) \text { alors } 1(\theta)=x^{*}{ }_{A}(\theta) f(\theta) \text { et } v(C, \theta) \leq v^{*}(\theta) \\
& \text {.si } u(C, \theta)<u(C, \theta) \text { alors } 1(\theta)=0
\end{aligned}
$$

On peut alors affirmer que, pour tout contrat $C$ on a:

$$
\mathrm{v}(\mathrm{C}, \theta) l(\theta) \leq \mathrm{v}^{*}(\theta) \mathrm{h}(\theta) \text { et donc } \Pi_{\mathrm{A}}\left(\mathrm{C}, \mathrm{C}^{*}\right) \leq \Pi_{\mathrm{A}}\left(\mathrm{C}^{*}, \mathrm{C}^{*}\right.
$$

Ces résultats nous montrent qu'un marché caractérisé par une asymétrie d'information entre offreurs et demandeurs peut être aussi efficace qu'un marché d'information parfaite. L'efficacité du marché dépend de la structure de la relation qui s'établit entre le principal et l'agent. Si l'information dont dispose l'agent n'affecte que son propre bien-être, autrement dit si le principal connaît le gain qu'il tire de l'échange avec l'agent, l'asymétrie d'information n'implique aucune perte de bien-être.

Ainsi, sur le marché de l'assurance, quand le principal ignore la probabilité d'accident de l'agent, la concurrence que se livrent les compagnies d'assurance n'est pas une condition suffisante pour que l'agent obtienne son bien-être maximum. En effet, la probabilité d'accident affecte l'espérance de profit qu'une compagnie d'assurance obtient quand elle assure un agent d'un type donné. Mais on pourrait imaginer que les probabilités d'accident des agents sont toutes identiques, et que certains agents subissent des dommages plus importants que d'autres. Les bas risques seraient alors les agents dont le dommage subi en cas d'accident est faible, les hauts risques ceux dont le dommage est le plus important. Avant accident, la compagnie d'assurance ignore le type de l'assuré, et elle ne peut observer le dommage subi après accident.

Dans ce cas, le type de l'agent n'affecte pas l'espérance de profit de sa compagnie d'assurance si celle-ci lui propose un échange spécifiant, comme chez RS, le paiement d'une prime et d'un montant remboursé en cas d'accident. Dès lors que la concurrence est suffisamment vive sur le marché de l'assurance, les agents obtiendront des contrats 
d'assurance parfaite, quel que soit leur type, et aucun d'entre eux ne souffrira de l'asymétrie d'information.

Un autre exemple pourrait être le cas où les agents different par leur aversion par rapport au risque: les probabilités d'accident sont identiques, mais les fonctions d'utilité des agents sont différentes, et sont bien-sûr une information privée.

Autrement dit, l'efficacité d'un tel marché dépend fondamentalement de la façon dont l'information connue de l'agent affecte le bien-être du principal. Dans la section suivante, on montre que l'efficacité du marché est liée à la structure d'information qui affecte l'échange des deux parties contractantes, et d'une façon assez fine.

\section{Section IV: Structure d'information et efficacité de l'équilibre RS}

Peut-on comparer l'issue de deux jeux RS selon la structure du jeu principal-agent de base?

Reprenons notre problème. Le modèle principal-agent de base est donné par:

- un ensemble d'échanges observables $Y$

- un ensemble de transferts monétaires possibles $T$

- deux fonctions de bien-être dépendant du type de l'agent $\mathrm{U}(\mathrm{y}, \mathrm{t}, \boldsymbol{\theta})$ et $\mathrm{V}(\mathrm{y}, \mathrm{t}, \boldsymbol{\theta})$, fonctions définies de $\mathrm{Y}^{*} \mathrm{~T}$ dans $\mathrm{R}$.

Supposons désormais qu'il existe un autre modèle principal-agent, $\mathrm{Z}, \mathrm{U}^{*}$ et $\mathrm{V}^{*}$, où:

. Z est un ensemble d'échanges observables.

. $\mathrm{U}^{*}(\mathrm{z}, \mathrm{t}, \boldsymbol{\theta})$, et $\mathrm{V}^{*}(\mathrm{z}, \mathrm{t}, \theta)$ sont des fonctions de bien-être définies de $\mathrm{Z}^{*} \mathrm{~T}$. dans $\mathrm{R}$.

Les problèmes sont comparables au sens suivant:

Pour $\theta$ et $\mathrm{t}$ donné, quel que soit $\mathrm{y} \in \mathrm{Y}$, il existe $\mathrm{z} \in \mathrm{Z}$ tel que:

$\mathrm{V}^{*}(\mathrm{z}, \mathrm{t}, \boldsymbol{\theta})=\mathrm{V}(\mathrm{y}, \mathrm{t}, \boldsymbol{\theta})$ et $\mathrm{U}(\mathrm{y}, \mathrm{t}, \boldsymbol{\theta})=\mathrm{U}^{*}(\mathrm{z}, \mathrm{t}, \boldsymbol{\theta})$

Quel que soit $\theta$ et $t$, quel que soit $z \in Z$, alors il existe $Y$ appartenant à y tel que:

$\mathrm{V}^{*}(\mathrm{z}, \mathrm{t}, \theta)=\mathrm{V}(\mathrm{y}, \mathrm{t}, \boldsymbol{\theta})$ et $\mathrm{U}(\mathrm{y}, \mathrm{t}, \theta)=\mathrm{U}^{*}(\mathrm{z}, \mathrm{t}, \boldsymbol{\theta})$

L'intuition de cette formalisation peut être décrite ainsi. Imaginons qu'un échange entre deux parties, un principal et un agent, soit représenté par un couple décision, transfert. Par exemple l'agent décide d'une action, a, et le principal verse un transfert à l'agent, $t$. Le bien-être de chaque partie dépend aussi d'un paramètre $\theta$ qu'ignore le principal et que connaît l'agent. Mais l'action n'est pas observable.Principal et agent ne peuvent signer un accord que sur la base de données observables et seul un signal $\mathrm{z}$ de l'échange est connu, 
où $\mathrm{z} \in \mathrm{Z}, \mathrm{Z}$ ensemble de signaux. Bien-sûr, il existe un lien entre l'action a et le signal $\mathrm{z}$. Ceci signifie qu'il existe une relation entre a et $z$, pour un $\theta$ donné, ou encore que l'action véritable est une fonction de $z$ et de $\theta$, soit $a=a(z, \theta)$. L'observation du signal $z$ donne aux parties contractantes un bien-être qui est fonction de $z$, soit $\mathrm{v}(\mathrm{z}, \mathrm{t}, \boldsymbol{\theta})$ pour le principal et $\mathrm{u}(\mathrm{z}, \mathrm{t}, \boldsymbol{\theta})$ pour l'agent, que l'on peut définir par:.

$$
\mathrm{u}(\mathrm{z}, \mathrm{t}, \boldsymbol{\theta})=\mathrm{U}_{\mathrm{A}}(\mathrm{a}(\mathrm{z}, \boldsymbol{\theta}), \mathrm{t}, \boldsymbol{\theta}) \text { et } \mathrm{v}(\mathrm{z}, \mathrm{t}, \boldsymbol{\theta})=\mathrm{U}_{\mathrm{P}}(\mathrm{a}(\mathrm{z}, \boldsymbol{\theta}), \mathrm{t}, \boldsymbol{\theta})
$$

$U_{A}$ et $U_{P}$ désigne le bien-être de chaque partie pour une action a et un transfert $t$. Mais bien entendu, il peut exister plusieurs technologies de signal. L'audit par exemple dans les entreprises est un moyen de contrôler les décisions des agents. Mais on peut aussi établir des contrats contingents à la quantité produite, ou à la qualité de la production... Dans cette section, la question posée est alors: existe-t-il des technologies de signaux plus performantes que d'autres ? La réponse que l'on peut apporter à cette question dépend bien-sûr du contexte économique, une technologie de signal sera par exemple plus performante sur un marché où les principaux sont très concurrencés que sur un marché sur lequel un principal exerce une position de monopole.

\section{Un exemple:}

La production d'une entreprise dépend de l'effort (a) et de la qualité $(\theta)$ de son directeur de la façon suivante $q=\theta a$. Le directeur subit un coût $C(a, \theta)=a^{2} / 2 \theta$ s'il prend la décision a et si sa qualité est $\theta$. La marge de l'entreprise, est aléatoire et son espérance est une fonction de $\theta, \mathrm{m}(\theta)$.

Les actionnaires ont deux possibilités pour inciter le directeur:

- observer l'effort a

- observer la quantité produite q.

Le bien-être des parties contractantes (actionnaires, directeur) s'écrit alors:

Pour un contrat contingent à l'effort $(a, t)$ :

$\mathrm{m}(\theta) \mathrm{a} \theta-\mathrm{t}$

$\mathrm{t}-\mathrm{a}^{2} / 2 \theta$

Pour un contrat contingent à la quantité $(\mathrm{q}, \mathrm{t})$ :

$\mathrm{m}(\theta) \mathrm{q}-\mathrm{t}$

$\mathrm{t}-\mathrm{q}^{2} / 2 \theta^{2}$

Si la concurrence que subissent les actionnaires ${ }^{3}$ sur le marché de l'emploi managérial est suffisamment vive, quelle structure d'information fournit l'utilité maximale au manager?

${ }^{3}$ Tous les paramètres de la relation sont pertinents pour un autre groupe d'actionnaires. 
On a vu dans la section précédente que toutes les structures de signal ne sont pas équivalentes. Dans cette section, on propose une définition de la précision de la structure d'information :

\section{Définition :}

Quel que soit $\theta_{\mathrm{i}}$,on note $\mathrm{z}\left(\mathrm{y}, \theta_{\mathrm{i}}\right) \in \mathrm{Z}$ l'observable dans la structure $\alpha^{*}$ qui vérifie: $\mathrm{V}^{*}\left(\mathrm{z}\left(\mathrm{y}, \theta_{\mathrm{i}}\right), \mathrm{t}, \theta_{\mathrm{i}}\right)=\mathrm{V}\left(\mathrm{y}, \mathrm{t}, \theta_{\mathrm{i}}\right)$ et $\mathrm{U}^{*}\left(\mathrm{z}\left(\mathrm{y}, \theta_{\mathrm{i}}\right), \mathrm{t}, \theta_{\mathrm{i}}\right)=\mathrm{U}\left(\mathrm{y}, \mathrm{t}, \theta_{\mathrm{i}}\right)$

La structure d'information $\alpha^{*}$ est plus précise que la structure $\alpha$ si pour tout $(y, t) \in \mathrm{Y}^{*} \mathrm{~T}$, on a:

$\operatorname{Inf}_{k} V\left(y, t, \theta_{1}\right) \leq V^{*}\left(z\left(y, \theta_{i}\right), t, \theta_{j}\right) \leq \sup _{k} V\left(y, t, \theta_{1}\right)$, pour tout $i$, tout $j$.

\section{Interprétation:}

Quand le principal ne connaît pas le type de l'agent, proposer un échange $(y, t)$ dans la structure de signal $\alpha$ lui donne un profit $V\left(y, t, \theta_{1}\right)$ si le type de l'agent est $\theta_{1}$ et $V\left(y, t, \theta_{2}\right)$ si le type de l'agent est $\theta_{2}$. Dès lors que ces expressions dépendent de $\theta$, son profit est une variable aléatoire ex ante (avant échange), qui prend la valeur $\mathrm{V}\left(\mathrm{y}, \mathrm{t}, \theta_{\mathfrak{i}}\right)$ avec la probabilité $\gamma_{i}$. De même pour la structure $\alpha^{*}$.

On ne peut comparer les deux structures d'information que pour des échanges qui permettent d'obtenir les mêmes biens-êtres. Si un échange $(y, t)$ permet à l'agent de type $\theta_{\mathrm{i}}$ d'obtenir un bien-être u et donne au principal une utilité v si le type de l'agent est $\theta_{\mathbf{i}}$, on sait qu'il existe un échange $(\mathrm{z}, \mathrm{t})^{4}$ dans la structure d'information $\alpha^{*}$, équivalent à l'échange $(y, t)$ dans la structure $\alpha$ si le type de l'agent est $\theta_{\mathrm{i}}$ : les niveaux de bien-être de l'agent et du principal sont identiques.

Mais le bien-être du principal est toujours, quelle que soit la structure de signal, aléatoire dès lors qu'il ignore le type de l'agent. Cette variable aléatoire prend les valeurs:

- dans la structure $\alpha, V\left(y, t, \theta_{i}\right)$ (avec la probabilité $\left.\gamma_{i}\right)$ et $V\left(y, t, \theta_{j}\right)\left(\gamma_{j}\right), i=1,2, j=1,2$

- dans la structure $\alpha^{*}, V^{*}\left(z, t, \theta_{i}\right)$ (avec la probabilité $\left.\gamma_{i}\right)$ et $V^{*}\left(z, t, \theta_{j}\right)\left(\gamma_{j}\right) i=1,2, j=1,2$

Peut-on comparer ces variables aléatoires ? La définition énoncée implique que pour $\theta_{i}$ donné, pour tout échange $(\mathrm{y}, \mathrm{t})$ dans la structure $\alpha$, alors on peut trouver un échange équivallent $(z, t)$ dans la structure $\alpha^{*}$, et celui-ci vérifie:

Il existe $k \in[0,1]$ tel que:

$\mathrm{V}^{*}\left(\mathrm{z}, \mathrm{t}, \theta_{\mathrm{i}}\right)-\mathrm{V}^{*}\left(\mathrm{z}, \mathrm{t}, \theta_{\mathrm{j}}\right)=\mathrm{k}\left\{\mathrm{V}\left(\mathrm{y}, \mathrm{t}, \theta_{\mathrm{i}}\right)-\mathrm{V}\left(\mathrm{y}, \mathrm{t}, \theta_{\mathrm{j}}\right)\right\}$

Ceci revient alors à dire que d'une certaine façon, le profit du principal dans la structure $\alpha$ est plus variable que le profit du principal dans la structure $\alpha^{*}$, et donc dans un certain sens que la structure $\alpha$ est plus "risquée" que la structure d'information $\alpha^{*}$.

\footnotetext{
${ }^{4}$ Le transfert, variable observable comune aux deux structures d'information, est identique.
} 
Une structure d'information donnée peut très bien avoir pour conséquence que le bienêtre du principal ne dépend plus du type de l'agent: on a alors $V^{*}\left(z, t, \theta_{1}\right)=V^{*}\left(z, t, \theta_{2}\right)$. L'information dans cette structure d'information est à valeurs privées. La variance du bien-être du principal est alors nulle.

Enfin remarquons que l'on a imposé que la structure $\alpha^{*}$ ne change pas le sens de la variation du profit par rapport au type de l'agent. Cette hypothèse est restrictive mais nécessaire pour obtenir nos résultats. L'agent le plus rentable pour un signal donné dans la structure $\alpha$ reste le plus rentable dans la structure $\alpha^{*}$ pour un signal équivalent ${ }^{5}$.

On suppose de plus que :

$\left(\mathrm{H}_{6}\right) \mathrm{Si}$, pour un transfert $\mathrm{t}$ donné, il existe $\mathrm{y} \in \mathrm{Y}$ et $\mathrm{y}^{\prime} \in \mathrm{Y}$ tel que l'agent d'un type donné préfêre y à $y^{\prime}$, alors le principal préfère $y^{\prime}$ à $y$ :

si $\mathrm{U}\left(\mathrm{y}, \mathrm{t}, \theta_{\mathrm{i}}\right)>\mathrm{U}\left(\mathrm{y}^{\prime}, \mathrm{t}, \theta_{\mathrm{i}}\right)$ alors $\mathrm{V}\left(\mathrm{y}, \mathrm{t}, \theta_{\mathrm{i}}\right)<\mathrm{V}\left(\mathrm{y}^{\prime}, \mathrm{t}, \theta_{\mathrm{i}}\right)$.

Cette hypothèse reflète le conflit d'intérêt existant entre le principal et l'agent.

Notons $P(\lambda)$ le programme:

$\operatorname{Max} \lambda \mathrm{U}\left(\mathrm{y}_{1}, \mathrm{t}_{1}, \theta_{1}\right)+(1-\lambda) \mathrm{U}\left(\mathrm{y}_{2}, \mathrm{t}_{2}, \theta_{2}\right)$

$\mathrm{y}_{1}, \mathrm{y}_{2}, \mathrm{t}_{1}, \mathrm{t}_{2}$

sc:

$\mathrm{U}\left(\mathrm{y}_{1}, \mathrm{t}_{1}, \theta_{1}\right) \geq \mathrm{U}\left(\mathrm{y}_{2}, \mathrm{t}_{2}, \theta_{1}\right)$

$\mathrm{U}\left(\mathrm{y}_{2}, \mathrm{t}_{2}, \theta_{2}\right) \geq \mathrm{U}\left(\mathrm{y}_{1}, \mathrm{t}_{1}, \theta_{2}\right)$

$\mathrm{V}\left(\mathrm{y}_{1}, \mathrm{t}_{1}, \theta_{1}\right) \geq 0$

$\mathrm{V}\left(\mathrm{y}_{2}, \mathrm{t}_{2}, \theta_{2}\right) \geq 0$

Et soit $\mathrm{P}^{*}(\lambda)$ le programme:

$\operatorname{Max} \lambda U^{*}\left(\mathrm{z}_{1}, \mathrm{t}_{1}, \theta_{1}\right)+(1-\lambda) \mathrm{U}^{*}\left(\mathrm{z}_{2}, \mathrm{t}_{2}, \theta_{2}\right)$

$\mathrm{z}_{1}, \mathrm{z}_{2}, \mathrm{t}_{1}, \mathrm{t}_{2}$

sc:

$\mathrm{U}^{*}\left(\mathrm{z}_{1}, \mathrm{t}_{1}, \theta_{1}\right) \geq \mathrm{U}^{*}\left(\mathrm{z}_{2}, \mathrm{t}_{2}, \theta_{1}\right)$

$\mathrm{U}^{*}\left(\mathrm{z}_{2}, \mathrm{t}_{2}, \theta_{2}\right) \geq \mathrm{U}^{*}\left(\mathrm{z}_{1}, \mathrm{t}_{1}, \theta_{2}\right)$

$\mathrm{V}^{*}\left(\mathrm{z}_{1}, \mathrm{t}_{1}, \theta_{1}\right) \geq 0$

$\mathrm{V}^{*}\left(\mathrm{z}_{2}, \mathrm{t}_{2}, \theta_{2}\right) \geq 0$

${ }^{5}$ On peut aisément vérifier que, dans l'exemple actionnaires manager, la structure d'information qui permet d'observer q est plus précise que celle qui permet d'observer $a$, dès lors que $m(\theta)$ est une fonction croissante de $\theta$. 


\section{Proposition 6:}

Si $\mathrm{H}_{6}$ est vraie, si la structure d'information $\alpha^{*}$ est plus précise que la structure $\alpha$, alors la solution du programme $\mathrm{P}^{*}(\lambda)$ donne un bien-être plus grand à l'agent quel que soit son type que la solution du programme $P(\lambda)$.

Le contrat RS est donc meilleur du point de vue de l'agent quand la structure d'information du principal est plus précise. Une structure d'information plus précise conduit à un équilibre RS plus performant du point de vue de l'efficacité.

Par contre, si le contrat RS est un équilibre pour la structure $\alpha$ (efficace au sens du second rang), il n'est pas évident que le contrat RS de la structure $\alpha^{*}$ soit lui aussi un équilibre pour la structure $\alpha^{*}$. Autrement dit, augmenter l'efficacité de l'équilibre ne signifie pas assurer l'existence de cet équilibre. Modifier la structure d'information modifie les contraintes d'incitations et permet certainement d'atteindre une meilleure solution de second rang. Le second rang rend maximum le bien-être de l'agent dans un ensemble de possibles et augmenter la précision de la structure d'information permet aussi d'augmenter l'ensemble des possibles du second rang. Un exemple est présenté en annexe 2, dans lequel on montre que le contrat RS est un équilibre du jeu pour une structure d'information donnée, alors que, pour une structure d'information plus précise au sens défini ici, le contrat RS n'est pas un équilibre du jeu.

\section{Exemple:}

Si $\mathrm{m}(\theta)$ est une fonction croissante de $\theta$, observer la quantité produite dans notre exemple actionnaires manager permet donc de donner au manager un bien-être plus grand quel que soit son type. On doit donc raisonnablement s'attendre à ce qu'elle soit choisie, si la concurrence que se livre les actionnaires pour ce directeur est suffisamment vive. Ceci ne signifie pas bien sûr que cette structure d'information sera choisie quel que soit l'environnement économique. Si les actionnaires sont en position de monopole face au manager, il est tout à fait possible que la structure d'information dans laquelle on observe l'effort soit préférée à celle dans laquelle on observe la quantité produite. 6

Preuve de la proposition 6:

Soit $\left(\mathrm{y}_{1} \mathrm{RS}_{, \mathrm{t}_{1}} \mathrm{RS}, \mathrm{y}_{2}{ }^{\mathrm{RS}}, \mathrm{t}_{2}{ }^{\mathrm{RS}}\right)$ la solution du programme $\mathrm{P}(\lambda)$ et $\left(\mathrm{z}_{1}{ }^{*}, \mathrm{t}_{1}{ }^{*}, \mathrm{z}_{2}{ }^{*}, \mathrm{t}_{2}{ }^{*}\right)$ celle du programme $\mathrm{P}^{*}(\lambda)$.

Supposons que $U\left(y_{1}{ }^{R S}, t_{1}{ }^{R S}, \theta_{1}\right)>U^{*}\left(z_{1}{ }^{*}, t_{1}{ }^{*}, \theta_{1}\right)$ et montrons que ceci est impossible.

\footnotetext{
${ }^{6}$ Un exemple numérique simple permet d'observer ceci: le manager doit obtenir 0 quel que soit son type et $\theta_{1}=2, \theta_{2}=1$.
} 
Soit $z_{i}=z\left(y_{i}{ }^{R S}, \theta_{i}\right)$.

Si le contrat $\left\{\left(z_{1}^{\prime}, t^{\prime}\right)\right.$ et $\left.\left(z_{2}^{\prime}, t_{2}^{\prime}\right)\right\}=\left\{\left(z_{1}, t_{1} R S\right),\left(z_{2}, t_{2} R S\right)\right\}$ satisfait les contraintes d'incitations de $\mathrm{P}^{*}$ :

$$
\begin{aligned}
& U^{*}\left(z_{1}{ }^{\prime}, t_{1}{ }^{\prime}, \theta_{1}\right) \geq U^{*}\left(z_{2}^{\prime}, t_{2}{ }^{\prime}, \theta_{1}\right) \\
& U^{*}\left(z_{2}^{\prime}, t_{2}^{\prime}, \theta_{2}\right) \geq U^{*}\left(z_{1}^{\prime}, t_{1}{ }^{\prime}, \theta_{2}\right)
\end{aligned}
$$

Ce que l'on cherche à montrer est prouvé, puisque

$$
\begin{aligned}
& V^{*}\left(z_{1}{ }^{\prime}, t_{1}{ }^{\prime}, \theta_{1}\right)=V\left(y_{1}{ }^{R S}, t_{1}{ }^{R S}, \theta_{1}\right) \geq 0 \\
& V^{*}\left(z_{2}{ }^{\prime}, t_{2}^{\prime}, \theta_{2}\right)=V\left(y_{2}{ }^{R S}, t_{2}{ }^{P}, \theta_{2}\right) \geq 0
\end{aligned}
$$

Supposons donc que l'une des deux contraintes d'incitations n'est pas vérifiée, soit:

$$
\mathrm{U}^{*}\left(\mathrm{z}_{1}, \mathrm{t}_{1}{ }^{\mathrm{RS}}, \theta_{1}\right)<\mathrm{U}^{*}\left(\mathrm{z}_{2}, \mathrm{t}_{2}{ }^{\mathrm{RS}}, \theta_{1}\right)
$$

On a alors $U^{*}\left(z_{2}, t_{2}{ }^{R S}, \theta_{1}\right)>U^{*}\left(z_{1}, t_{1}{ }^{R S}, \theta_{1}\right)=U\left(y_{1}{ }^{R S}, t_{1}{ }^{R S}, \theta_{1}\right) \geq U\left(y_{2}{ }^{R S}, t_{2}{ }^{R S}, \theta_{1}\right)$

Donc $\mathrm{U}^{*}\left(\mathrm{z}_{2}, \mathrm{t}_{2}{ }^{\mathrm{RS}}, \theta_{1}\right)>\mathrm{U}\left(\mathrm{y}_{2}{ }^{\mathrm{RS}}, \mathrm{t}_{2}{ }^{\mathrm{RS}}, \theta_{1}\right)$

Par conséquent, si l'on note y l'échange de $\mathrm{Y}$ qui vérifie:

$\mathrm{U}^{*}\left(\mathrm{z}_{2}, \mathrm{t}_{2} \mathrm{RS}, \theta_{1}\right)=\mathrm{U}\left(\mathrm{y}, \mathrm{t}_{2}{ }^{\mathrm{RS}}, \theta_{1}\right)$ et $\mathrm{V}^{*}\left(\mathrm{z}_{2}, \mathrm{t}_{2}{ }^{\mathrm{RS}}, \theta_{1}\right)=\mathrm{V}\left(\mathrm{y}, \mathrm{t}_{2}{ }^{\mathrm{RS}}, \theta_{1}\right)$,

y vérifie:

$\mathrm{U}\left(\mathrm{y}, \mathrm{t}_{2}{ }^{\mathrm{RS}}, \theta_{1}\right)>\mathrm{U}\left(\mathrm{y}_{2}{ }^{\mathrm{RS}}, \mathrm{t}_{2}{ }^{\mathrm{RS}}, \theta_{1}\right)$, donc

$\mathrm{V}^{*}\left(\mathrm{z}_{2}, \mathrm{t}_{2}{ }^{\mathrm{RS}}, \theta_{1}\right)=\mathrm{V}\left(\mathrm{y}, \mathrm{t}_{2}{ }^{\mathrm{RS}}, \theta_{1}\right)<\mathrm{V}\left(\mathrm{y}_{2}{ }^{\mathrm{RS}}, \mathrm{t}_{2}{ }^{\mathrm{RS}}, \theta_{1}\right)$.

En appliquant le fait que la structure d'information $\alpha^{*}$ est plus précise que la structure d'information $\alpha$, on a : $\mathrm{V}\left(\mathrm{y}_{2}{ }^{\mathrm{RS}}, \mathrm{t}_{2}{ }^{\mathrm{RS}}, \theta_{1}\right)>\mathrm{V}^{*}\left(\mathrm{z}_{2}, \mathrm{t}_{2}{ }^{\mathrm{RS}}, \theta_{1}\right) \geq \mathrm{V}^{*}\left(\mathrm{z}_{2}, \mathrm{t}_{2}{ }^{\mathrm{RS}}, \theta_{2}\right) \geq 0$.

Mais alors le contrat $\left\{\left(\mathrm{z}_{1}{ }^{\prime}, \mathrm{t}_{1}{ }^{\prime}\right),\left(\mathrm{z}_{2}{ }^{\prime}, \mathrm{t}_{2}^{\prime}\right)\right\}=\left\{\left(\mathrm{z}_{2}, \mathrm{t}_{2} \mathrm{RS}\right),\left(\mathrm{z}_{2}, \mathrm{t}_{2} \mathrm{RS}\right)\right\}$ satisfait les contraintes d'incitations, donne à l'agent quel que soit son son type un bien-être plus grand que le contrat RS, et dégage pour le principal un bien-être positif ou nul quel que soit le type de l'agent.

QED

\section{Section V Conclusion}

Il apparaît donc au regard des résultats de la section IV que plus la structure d'information du principal est précise, plus le bien-être qu'obtient l'agent quand on lui propose le contrat de Rotschild et Stiglitz est élevé, quelque soit son type. Autrement dit, l'issue du processus concurrentiel qui tend à rendre maximum le bien-être de l'agent n'est pas indépendant de la structure d'information disponible au sein de la relation principal-agent. Supposer que le bien-être du principal est indépendant du type de l'agent est le cas limite cette étude, et l'agent dans ce cas obtient, à l'équilibre du jeu de concurrence entre les deux principaux, son bien-être d'information parfaite. 


\section{ANNEXE 1}

\section{Proposition 1:}

Si $\mathrm{H}_{1}$ est vérifiée, $\mathrm{RS}$ est une proposition d'équilibre du jeu si et seulement si P est vraie.

\section{Preuve de la proposition:}

La preuve est conduite en deux temps:

i) si P est vraie, alors RS est une proposition d'équilibre du jeu.

ii) si $P$ est fausse, RS n'est pas une proposition d'équilibre du jeu.

i) si P est vraie, alors $R S$ est une proposition d'équilibre du jeu.

Remarquons que si $P$ est vraie alors $V\left(y_{i}{ }^{R S}, \theta_{i}\right)=0, i=1,2$

Soit $\mathrm{x}_{\mathrm{K}}{ }^{*}\left(\theta_{\mathrm{i}}\right), \mathrm{i}=1,2$, la règle de décision de l'agent quand les deux principaux proposent le contrat RS.

Supposons que l'un des principaux (par exemple A) propose le contrat RS. Quel profit peut réaliser le principal $\mathrm{B}$ si A propose le contrat RS ?

Notons $C_{B}$ la proposition de $B: C_{B}=\left\{y_{1}, y_{2}, \ldots, y_{n}\right\}$. Soit $y_{i}$ l'échange de ce contrat préféré par l'agent de type $\theta_{i}, i=1,2$. Soit $x_{B}\left(\theta_{i}\right)$ la règle de décision de l'agent :

- si $\mathrm{U}\left(\mathrm{y}_{\mathrm{i}}, \theta_{\mathrm{i}}\right)>\mathrm{U}\left(\mathrm{y}_{\mathrm{i}}^{\mathrm{RS}}, \theta_{\mathrm{i}}\right), \mathrm{x}_{\mathrm{B}}\left(\theta_{\mathrm{i}}\right)=1$

- si $\mathrm{U}\left(\mathrm{y}_{\mathrm{i}}, \theta_{\mathrm{i}}\right)=\mathrm{U}\left(\mathrm{y}_{\mathrm{i}}^{\mathrm{RS}}, \theta_{\mathrm{i}}\right), \mathrm{x}_{\mathrm{B}}\left(\theta_{\mathrm{i}}\right)=\mathrm{x}_{\mathrm{B}}{ }^{*}\left(\theta_{\mathrm{i}}\right)$

- si $U\left(y_{i}, \theta_{i}\right)<U\left(y_{i}{ }^{R S}, \theta_{i}\right), x_{B}\left(\theta_{i}\right)=0$

Plusieurs cas sont possibles:

- si $U\left(y_{i}, \theta_{i}\right)<U\left(y_{i} R S, \theta_{i}\right), i=1,2$, alors en proposant $C_{B}$, le principal B réalise un profit nul. En effet, l'agent quel que soit son type préfère le contrat du principal $A$. Il préfère donc ( faiblement) proposer le contrat $\mathrm{RS}$ au contrat $\mathrm{C}_{\mathrm{B}}$.

- si $\mathrm{U}\left(\mathrm{y}_{\mathrm{i}}, \theta_{\mathrm{i}}\right)>\mathrm{U}\left(\mathrm{y}_{\mathrm{i}}^{\mathrm{RS}}, \theta_{\mathrm{i}}\right), \mathrm{i}=1,2$, alors le principal $\mathrm{B}$ réalise un profit :

$\gamma_{1} V\left(y_{1}, \theta_{1}\right)+\gamma_{2} V\left(y_{2}, \theta_{2}\right) \leq 0$ d'après la propriété $(P)$. Proposer $C_{B}$ pour le principal $B$ n'est pas plus avantageux que proposer le contrat RS.

- si $\mathrm{U}\left(\mathrm{y}_{1}, \theta_{1}\right)>\mathrm{U}\left(\mathrm{y}_{1}^{\mathrm{RS}}, \theta_{1}\right)$ et $\mathrm{U}\left(\mathrm{y}_{2}, \theta_{2}\right) \leq \mathrm{U}\left(\mathrm{y}_{2}{ }^{\mathrm{RS}}, \theta_{2}\right)$, alors on a $\mathrm{V}\left(\mathrm{y}_{1}, \theta_{1}\right)<0$.

En effet, supposons que $\mathrm{V}\left(\mathrm{y}_{1}, \theta_{1}\right) \geq 0$.

Comme les contraintes suivantes sont satisfaites:

$\mathrm{U}\left(\mathrm{y}_{1}, \theta_{1}\right) \geq \mathrm{U}\left(\mathrm{y}_{2}, \theta_{1}\right)$

$\mathrm{U}\left(\mathrm{y}_{2}, \theta_{2}\right) \geq \mathrm{U}\left(\mathrm{y}_{1}, \theta_{2}\right)$

par définition des échange $\left(\mathrm{y}_{1}, \mathrm{y}_{2}\right)$, on a aussi: 
$\mathrm{U}\left(\mathrm{y}_{1}, \theta_{1}\right)>\mathrm{U}\left(\mathrm{y}_{1}{ }^{\mathrm{RS}}, \theta_{1}\right) \geq \mathrm{U}\left(\mathrm{y}_{2}{ }^{\mathrm{RS}}, \theta_{1}\right)$

$\mathrm{U}\left(\mathrm{y}_{2}{ }^{\mathrm{RS}}, \theta_{2}\right) \geq \mathrm{U}\left(\mathrm{y}_{2}, \theta_{2}\right) \geq \mathrm{U}\left(\mathrm{y}_{1}, \theta_{2}\right)$

Mais alors si $\mathrm{V}\left(\mathrm{y}_{1}, \theta_{1}\right) \geq 0,\left(\mathrm{y}_{1} \mathrm{RS}, \mathrm{y}_{2} \mathrm{RS}\right)$ ne peut être la solution du programme $\mathrm{RS}$, ce qui est impossible.

. Si U $\left(\mathrm{y}_{2}, \theta_{2}\right)<U\left(\mathrm{y}_{2} \mathrm{RS}, \theta_{2}\right)$ alors le principal B réalise un profit $\gamma_{1} \mathrm{~V}\left(\mathrm{y}_{1}, \theta_{1}\right)<0$ et $\mathrm{C}_{\mathrm{B}}$ ne peut être une proposition d'équilibre.

. Si U $\left(\mathrm{y}_{2}, \theta_{2}\right)=\mathrm{U}\left(\mathrm{y}_{2}{ }^{\mathrm{RS}}, \theta_{2}\right)$, on a, d'après la propriété $(\mathrm{P})$ :

$\gamma_{1} \mathrm{~V}\left(\mathrm{y}_{1}, \theta_{1}\right)+\gamma_{2} \mathrm{~V}\left(\mathrm{y}_{2}, \theta_{2}\right) \leq 0$

Or en proposant un tel contrat, le principal $B$ obtient un profit: $\gamma_{1} \mathrm{~V}\left(\mathrm{y}_{1}, \theta_{1}\right)+\gamma_{2} \mathrm{xB}_{\mathrm{B}}{ }^{*}\left(\theta_{2}\right) \mathrm{V}\left(\mathrm{y}_{2}, \theta_{2}\right) \leq 0$ dès lors que $\mathrm{x}_{\mathrm{B}}{ }^{*}\left(\theta_{2}\right)$ qui désigne la probabilité pour que l'agent quand son type est $\theta_{2}$ choisisse l'échange du principal $B$ est comprise entre 0 et 1. Le principal $B$ préfere ( faiblement) proposer le contrat $R S$ plutôt que $C_{B}$.

- Il nous reste à étudier le cas où $\mathrm{U}\left(\mathrm{y}_{1}, \theta_{1}\right)=\mathrm{U}\left(\mathrm{y}_{1}{ }^{\mathrm{RS}}, \theta_{1}\right)$ et $\mathrm{U}\left(\mathrm{y}_{2}, \theta_{2}\right)<\mathrm{U}\left(\mathrm{y}_{2}{ }^{\mathrm{RS}}, \theta_{2}\right)$. Par définition du contrat $\mathrm{RS}$, on sait alors que $\mathrm{V}\left(\mathrm{y}_{1}, \theta_{1}\right) \leq \mathrm{V}\left(\mathrm{y}_{1} \mathrm{RS}, \theta_{1}\right)=0$

Pour une stratégie de l'agent quand celui ci est indifférent entre les contrats des deux principaux, donnée, $\mathrm{x}_{\mathrm{B}}{ }^{*}\left(\theta_{1}\right)$ et $\mathrm{x}_{\mathrm{B}}{ }^{*}\left(\theta_{2}\right)$, on a:

$\gamma_{1} \mathrm{x}_{\mathrm{B}}{ }^{*}\left(\theta_{1}\right) \mathrm{V}\left(\mathrm{y}_{1}, \theta_{1}\right) \leq \gamma_{1} \mathrm{x}_{\mathrm{B}}{ }^{*}\left(\theta_{1}\right) \mathrm{V}\left(\mathrm{y}_{1}{ }^{\mathrm{RS}}, \theta_{1}\right)+\gamma_{2} \mathrm{x}_{\mathrm{B}}{ }^{*}\left(\theta_{2}\right) \mathrm{V}\left(\mathrm{y}_{2}{ }^{\mathrm{RS}}, \theta_{2}\right)$

Par conséquent le principal $B$ préfere proposer le contrat RS plutôt que $C_{B}$.

ii) Si P est fausse, RS n'est pas une proposition d'équilibre du jeu.

Supposons que $P$ soit fausse et que RS soit une proposition d'équilibre du jeu. Dire que RS est une proposition d'équilibre du jeu signifie que chaque échange de RS est choisi avec une probabilité non nulle à l'équilibre. Si l'on note $C^{*}=\left\{y_{1}, y_{2}, y_{3}, y_{4} \ldots\right\}$ l'ensemble des échanges offerts à l'équilibre et choisis par l'agent avec une probabilité non nulle, alors on $a: y_{i} R S \in \operatorname{Arg} \max _{C}^{*} U\left(y, \theta_{i}\right), i=1,2$ ce qui signifie que pour tout $y \in$ $\mathrm{C}^{*}$, alors $\mathrm{U}\left(\mathrm{y}, \theta_{\mathrm{i}}\right) \leq \mathrm{U}\left(\mathrm{y}_{\mathrm{i}}^{\mathrm{RS}}, \theta_{\mathrm{i}}\right)$.

Si P est fausse, et si RS est proposé à l'équilibre alors on est sûr que l'espérance de bienêtre de chaque principal est strictement positive à l'équilibre. En effet, si P est fausse notons $\left(\mathrm{y}_{1}, \mathrm{y}_{2}\right) \in \mathrm{Y}^{2}$ et satisfaisant IC qui vérifie:

$\mathrm{U}\left(\mathrm{y}_{\mathbf{i}}, \theta_{\mathrm{i}}\right) \geq \mathrm{U}\left(\mathrm{y}_{\mathrm{i}}^{\mathrm{RS}}, \theta_{\mathrm{i}}\right)$

$\gamma_{1} \mathrm{~V}\left(\mathrm{y}_{1}, \theta_{1}\right)+\gamma_{2} \mathrm{~V}\left(\mathrm{y}_{2}, \theta_{2}\right)>0$

On sait alors $\left(\mathrm{H}_{1}\right)$ qu'il existe $\left(\mathrm{y}^{\prime} 1, \mathrm{y}^{\prime} 2\right) \in \mathrm{Y}^{2}$ et satisfaisant IC tel que:

$\mathrm{U}\left(\mathrm{y}^{\prime}{ }_{\mathrm{i}}, \theta_{\mathrm{i}}\right)>\mathrm{U}\left(\mathrm{y}_{\mathrm{i}}, \theta_{\mathrm{i}}\right), \mathrm{i}=1,2$

$\gamma_{1} \mathrm{~V}\left(\mathrm{y}_{1}^{\prime}, \theta_{1}\right)+\gamma_{2} \mathrm{~V}\left(\mathrm{y}_{2}^{\prime}, \theta_{2}\right) \geq \gamma_{1} \mathrm{~V}\left(\mathrm{y}_{1}, \theta_{1}\right)+\gamma_{2} \mathrm{~V}\left(\mathrm{y}_{2}, \theta_{2}\right)-\varepsilon>0$ 
Or, si un tel contrat est proposé par l'un des principaux, l'agent quel que soit son type choisit ce contrat. Par conséquent, chaque principal réalise un profit strictement positif à l'équilibre.

On est donc sûr que l'un des échanges d'équilibre réalise un profit strictement positif. Soit $\mathrm{y}_{1 \mathrm{~A}}$ cet échange, $\mathrm{V}\left(\mathrm{y}_{1 \mathrm{~A}}, \theta_{1}\right)>0$. Supposons de plus que $\mathrm{V}\left(\mathrm{y}_{1 \mathrm{~A}}, \theta_{1}\right) \geq \mathrm{V}\left(\mathrm{y}_{1 \mathrm{~B}}, \theta_{1}\right)$. Trois cas sont successivement étudiés, et l'on montre que ceci est impossible à l'équilibre.

i) $x_{A}\left(\theta_{1}\right)>0$ et $x_{B}\left(\theta_{2}\right)>0$

$\left(\mathrm{y}_{1 \mathrm{~A}}, \mathrm{y}_{2 \mathrm{~B}}\right)$ satisfait IC

On peut trouver $\left(\mathrm{y}_{1}, \mathrm{y}_{2}\right) \in \mathrm{Y}^{2}$, satisfaisant IC, et tel qu'il existe $\varepsilon$ petit avec:

$\mathrm{U}\left(\mathrm{y}_{1}, \theta_{1}\right)>\mathrm{U}\left(\mathrm{y}_{1 \mathrm{~A}}, \theta_{1}\right), \mathrm{U}\left(\mathrm{y}_{2}, \theta_{2}\right)>\mathrm{U}\left(\mathrm{y}_{2 \mathrm{~B}}, \theta_{2}\right)$ et $\gamma_{1} \mathrm{~V}\left(\mathrm{y}_{1}, \theta_{1}\right)+\gamma_{2} \mathrm{~V}\left(\mathrm{y}_{2}, \theta_{2}\right) \geq \gamma_{1} \mathrm{~V}\left(\mathrm{y}_{1} \mathrm{~A}, \theta_{1}\right)+\gamma_{2} \mathrm{~V}\left(\mathrm{y}_{2 \mathrm{~B}}, \theta_{2}\right)-\varepsilon$

$>\gamma_{1} \mathrm{x}_{\mathrm{B}}\left(\theta_{1}\right) \mathrm{V}\left(\mathrm{y}_{1 \mathrm{~B}}, \theta_{1}\right) \gamma_{2} \mathrm{x}_{\mathrm{B}}\left(\theta_{2}\right) \mathrm{V}\left(\mathrm{y}_{2 \mathrm{~B}}, \theta_{2}\right)$ avec $\mathrm{V}\left(\mathrm{y}_{1 \mathrm{~A}}, \theta_{1}\right)>0, \mathrm{x}_{\mathrm{B}}\left(\theta_{1}\right)<1$ et $\mathrm{V}\left(\mathrm{y}_{1 \mathrm{~A}}, \theta_{1}\right) \geq \mathrm{V}\left(\mathrm{y}_{1 \mathrm{~B}}, \theta_{1}\right)$. $\left(\mathrm{y}_{1 \mathrm{~B}}, \mathrm{y}_{2 \mathrm{~B}}\right)$ ne peut être une proposition d'équilibre pour le principal $B$.

ii) $\mathrm{x}_{\mathrm{A}}\left(\theta_{1}\right)>0$ et $\mathrm{x}_{\mathrm{B}}\left(\theta_{2}\right)=0, \mathrm{~V}\left(\mathrm{y}_{1 \mathrm{~B}}, \theta_{1}\right)$ et $\mathrm{x}_{\mathrm{B}}\left(\theta_{1}\right)>0$.

On peut trouver $\left(\mathrm{y}_{1}, \mathrm{y}_{2}\right) \in \mathrm{Y}^{2}$, satisfaisant IC, et tel qu'il existe $\varepsilon$ petit avec:

$\mathrm{U}\left(\mathrm{y}_{1}, \theta_{1}\right)>\mathrm{U}\left(\mathrm{y}_{1 \mathrm{~A}}, \theta_{1}\right), \mathrm{U}\left(\mathrm{y}_{2}, \theta_{2}\right)>\mathrm{U}\left(\mathrm{y}_{2 \mathrm{~A}}, \theta_{2}\right) \geq \mathrm{U}\left(\mathrm{y}_{2 \mathrm{~B}}, \theta_{2}\right)$ et $\gamma_{1} \mathrm{~V}\left(\mathrm{y}_{1}, \theta_{1}\right)+\gamma_{2} \mathrm{~V}\left(\mathrm{y}_{2}, \theta_{2}\right) \geq \gamma_{1} \mathrm{~V}\left(\mathrm{y}_{1 \mathrm{~A}}, \theta_{1}\right)+\gamma_{2} \mathrm{~V}\left(\mathrm{y}_{2 \mathrm{~A}}, \theta_{2}\right)-\varepsilon$ $>\gamma_{1} \mathrm{x}_{\mathrm{A}}\left(\theta_{1}\right) \mathrm{V}\left(\mathrm{y}_{1 \mathrm{~A}}, \theta_{1}\right)+\gamma_{2} \mathrm{~V}\left(\mathrm{y}_{2 \mathrm{~A}}, \theta_{2}\right)$ avec $\mathrm{V}\left(\mathrm{y}_{1 \mathrm{~A}}, \theta_{1}\right)>0$.

$\left(\mathrm{y}_{1 \mathrm{~A}}, \mathrm{y}_{2} \mathrm{~A}\right)$ ne peut être une proposition d'équilibre pour le principal $\mathrm{A}$.

iii) $\mathrm{x}_{\mathrm{A}}\left(\theta_{1}\right)=0, \mathrm{x}_{\mathrm{A}}\left(\theta_{2}\right) \mathrm{V}\left(\mathrm{y}_{2 \mathrm{~A}}, \theta_{2}\right)>0$

. Si V $\left(\mathrm{y}_{1 \mathrm{~B}}, \theta_{1}\right)=0$, alors $\mathrm{x}_{\mathrm{B}}\left(\theta_{2}\right) \mathrm{V}\left(\mathrm{y}_{2 \mathrm{~B}}, \theta_{2}\right)>0$

$\left(\mathrm{y}_{1 \mathrm{~B}}, \mathrm{y}_{2 \mathrm{~A}}\right)$ satisfait IC et $\mathrm{B}$ a intérêt à dévier en proposant $\left(\mathrm{y}_{1}, \mathrm{y}_{2}\right)$ satisfaisant $\mathrm{CI}$ et tel que:

$\mathrm{U}\left(\mathrm{y}_{\mathrm{i}}, \theta_{\mathrm{i}}\right)>\mathrm{U}\left(\mathrm{y}_{\mathrm{iB}}, \theta_{\mathrm{i}}\right) \geq \mathrm{U}\left(\mathrm{y}_{\mathrm{iA}}, \theta_{\mathrm{i}}\right)$

$\gamma_{1} \mathrm{~V}\left(\mathrm{y}_{1}, \theta_{1}\right)+\gamma_{2} \mathrm{~V}\left(\mathrm{y}_{2}, \theta_{2}\right) \geq \gamma_{1} \mathrm{~V}\left(\mathrm{y}_{1 \mathrm{~B}}, \theta_{1}\right)+\gamma_{2} \mathrm{~V}\left(\mathrm{y}_{2 \mathrm{~B}}, \theta_{2}\right)-\varepsilon>\gamma_{2} \mathrm{x}_{\mathrm{B}}\left(\theta_{2}\right) \mathrm{V}\left(\mathrm{y}_{2 \mathrm{~B}}, \theta_{2}\right)$

. Si $\mathrm{V}\left(\mathrm{y}_{1 \mathrm{~B}}, \theta_{1}\right)>0,\left(\mathrm{y}_{1 \mathrm{~B}}, \mathrm{y}_{2} \mathrm{~A}\right)$ satisfait IC et $\mathrm{A}$ a intérêt à dévier en proposant $\left(\mathrm{y}_{1}, \mathrm{y}_{2}\right)$ satisfaisant $\mathrm{CI}$ et tel que:

$\mathrm{U}\left(\mathrm{y}_{1}, \theta_{1}\right)>\mathrm{U}\left(\mathrm{y}_{1 \mathrm{~B}}, \theta_{1}\right), \mathrm{U}\left(\mathrm{y}_{2}, \theta_{2}\right)>\mathrm{U}\left(\mathrm{y}_{2 \mathrm{~A}}, \theta_{2}\right) \geq \mathrm{U}\left(\mathrm{y}_{2 \mathrm{~B}}, \theta_{2}\right)$

$\gamma_{1} \mathrm{~V}\left(\mathrm{y}_{1}, \theta_{1}\right)+\gamma_{2} \mathrm{~V}\left(\mathrm{y}_{2}, \theta_{2}\right) \geq \gamma_{1} \mathrm{~V}\left(\mathrm{y}_{1 \mathrm{~B}}, \theta_{1}\right)+\gamma_{2} \mathrm{~V}\left(\mathrm{y}_{2 \mathrm{~A}}, \theta_{2}\right)-\varepsilon>\gamma_{2} \mathrm{x}_{\mathrm{A}}\left(\theta_{2}\right) \mathrm{V}\left(\mathrm{y}_{2 \mathrm{~A}}, \theta_{2}\right)$

QED 


\section{ANNEXE 2:}

Dans cette annexe, on démontre la proposition 4.

Si $\mathrm{H}_{1}$ est vraie, et si $\mathrm{U}$ et $\mathrm{V}$ sont des fonctions concaves de $\mathrm{y} \in \mathrm{Y}$, et s'il existe un échange y tel que $\mathrm{V}(\mathrm{y})>\operatorname{Max}\left(\mathrm{v}^{*}\left(\theta_{1}\right), \mathrm{v}^{*}\left(\theta_{2}\right)\right)$, si y est un échange choisi à l'équilibre par l'agent $\theta_{i}$, alors $V(y)=v^{*}\left(\theta_{i}\right)$ et $U\left(y, \theta_{i}\right)=u^{*}\left(\theta_{i}\right), i=1,2$

NOTATIONS de l'annexe:

On note $\left(C_{A}, C_{B}\right)$ les contrats proposés à l'équilibre par le principal $A$ et le principal $B$. yiK désigne l'échange du contrat $C_{K}$ préféré par l'agent si son type est $\theta_{i}, i=1,2, K=A$, B.

$y_{1 K}$ et $y_{2 K}$ peuvent bien sûr être confondus. Par définition on a donc: $U\left(y_{i K}, \theta_{i}\right) \geq$ $\mathrm{U}\left(\mathrm{y}_{\mathrm{jK}}, \theta_{\mathrm{j}}\right)$.

On note $\mathrm{x}_{\mathrm{K}}\left(\theta_{\mathrm{i}}\right) \in[0,1]$ la probabilité pour que l'agent si son type est $\theta_{\mathbf{i}}$ choisisse le contrat du principal $\mathrm{K}$.

L'espérance de bien-être d'équilibre du principal $K, K=A, B$ peut être écrit:

$\Pi_{K}\left(C_{A}, C_{B}\right)=\gamma_{1} x_{K}\left(\theta_{1}\right) V\left(y_{1 K}\right)+\gamma_{2} x_{K}\left(\theta_{2}\right) V\left(y_{2 K}\right)$

Rapellons que $\left(C_{A}, C_{B}\right)$ est un équilibre de Nash si

$$
\begin{aligned}
& \Pi_{A}\left(C_{A}, C_{B}\right) \geq \Pi_{A}\left(C, C_{B}\right) \text { pour tout } C \in Y^{2} \\
& \Pi_{B}\left(C_{A}, C_{B}\right) \geq \Pi_{B}\left(C_{A}, C\right) \text { pour tout } C \in Y^{2}
\end{aligned}
$$

Remarque 1:

Si $\mathrm{H}_{1}$ est vraie, $\mathrm{v}^{*}\left(\theta_{\mathrm{i}}\right)=0, \mathrm{i}=1,2$.

Remarque 2:

Si $v\left(C_{A}, \theta_{1}\right)<v^{*}\left(\theta_{1}\right)$ alors $x_{A}\left(\theta_{1}\right)=0$

\section{Preuve:}

Supposons que $v\left(C_{A}, \theta_{1}\right)<v^{*}\left(\theta_{1}\right)=0$ et $x_{A}\left(\theta_{1}\right)>0$.

Si $x_{A}\left(\theta_{2}\right) v\left(C_{A}, \theta_{2}\right) \leq 0$, alors $A$ en offrant $C^{*}$ est sûr d'obtenir un profit nul. Il dévie.

Si $x_{A}\left(\theta_{2}\right) v\left(C_{A}, \theta_{2}\right)>0, u\left(C_{A}, \theta_{2}\right) \geq u\left(C_{B}, \theta_{2}\right)$

Si A offre le contrat $C=\left\{\lambda y^{*}\left(\theta_{2}\right)+(1-\lambda) y_{2} A\right\}$, avec $1>\lambda>1, u\left(C, \theta_{2}\right)>u\left(C_{B}, \theta_{2}\right)$ et $A$ réalise un profit plus grand que:

$\gamma_{2} \lambda v\left(C_{A}, \theta_{2}\right)>\gamma_{1} x_{A}\left(\theta_{1}\right) v\left(C_{A}, \theta_{1}\right)+\gamma_{2} x_{B}\left(\theta_{2}\right) v\left(C_{A}, \theta_{2}\right)$ dès lors que 
$\lambda>\mathrm{x}_{\mathrm{B}}\left(\theta_{2}\right)+\frac{\gamma_{1} \mathrm{x}_{\mathrm{A}}\left(\theta_{1}\right) \mathrm{v}\left(\mathrm{C}_{\mathrm{A}}, \theta_{1}\right)}{\gamma_{2} \mathrm{v}\left(\mathrm{C}_{\mathrm{A}}, \theta_{2}\right)}$

Or cette expression est strictement plus petite que 1.QED

\section{Remarque 3:}

Si $u\left(C_{A}, \theta_{1}\right)<u^{*}\left(\theta_{1}\right)$, alors le profit d'équilibre de $B$ est strictement positif.

\section{Preuve:}

Il existe un contrat $C=\left\{\lambda y+(1-\lambda) y^{*}\left(\theta_{1}\right) ; \lambda y+(1-\lambda) y^{*}\left(\theta_{1}\right)\right\}$ tel que:

si $\lambda<1, v\left(C, \theta_{1}\right)>0$

$u\left(C, \theta_{1}\right)>u\left(C_{A}, \theta_{1}\right)$, pour un $\lambda$ donné $\lambda<\frac{u^{*}\left(\theta_{1}\right)-u\left(C_{A}, \theta_{1}\right)}{u^{*}\left(\theta_{1}\right)-u\left(y, \theta_{1}\right)}$

En offrant $C, B$ est sûr d'obtenir un profit strictement positif.

\section{Remarque 4:}

Si $\mathrm{u}\left(\mathrm{C}_{\mathrm{A}}, \theta_{1}\right)<\mathrm{u}^{*}\left(\theta_{1}\right), \mathrm{x}_{\mathrm{A}}\left(\theta_{1}\right)=0$

\section{Preuve:}

Supposons que $\mathrm{u}\left(\mathrm{C}_{\mathrm{A}}, \theta_{1}\right)<\mathrm{u}^{*}\left(\theta_{1}\right)$ et $\mathrm{x}_{\mathrm{A}}\left(\theta_{1}\right)>0$.

On sait que le profit d'équilibre de $B$ est strictement positif:

$\gamma_{1} \mathrm{x}_{\mathrm{B}}\left(\theta_{1}\right) \mathrm{v}\left(\mathrm{C}_{\mathrm{B}}, \theta_{1}\right)+\gamma_{2} \mathrm{x}_{\mathrm{B}}\left(\theta_{2}\right) \mathrm{v}\left(\mathrm{C}_{\mathrm{B}}, \theta_{2}\right)>0$

i) si $x_{B}\left(\theta_{2}\right) v\left(C_{B}, \theta_{2}\right)=0$, alors $u\left(C_{B}, \theta_{1}\right)=u\left(C_{A}, \theta_{1}\right)$.

Si B propose le contrat $\left\{\lambda y_{1 B}+(1-\lambda) y^{*}\left(\theta_{1}\right), \lambda y_{1 B}+(1-\lambda) y^{*}\left(\theta_{1}\right)\right\}$ avec $1>\lambda>0$, il obtient un profit plus grand que $\gamma_{1} \lambda \mathrm{v}\left(C_{B}, \theta_{1}\right)>\gamma_{1} \mathrm{x}_{B}\left(\theta_{1}\right) \mathrm{v}\left(C_{B}, \theta_{1}\right)$ dés lors que $\lambda>$ $\mathbf{x}_{\mathbf{B}}\left(\theta_{1}\right)$.

ii) $\sin x_{B}\left(\theta_{2}\right) v\left(C_{B}, \theta_{2}\right)>0$

a) $\mathrm{x}_{B}\left(\theta_{1}\right) \mathrm{v}\left(\mathrm{C}_{\mathrm{B}}, \theta_{1}\right)>0$, alors $\mathrm{u}\left(\mathrm{C}_{\mathrm{A}}, \theta_{1}\right)=\mathrm{u}\left(\mathrm{C}_{\mathrm{B}}, \theta_{1}\right)$ et $\left(\mathrm{y}_{1 \mathrm{~A}}, \mathrm{y}_{2 \mathrm{~B}}\right)$ satisfait IC.

Il existe $\left\{\mathrm{y}_{1}, \mathrm{y}_{2}\right\}$ satisfaisant IC tel que:

$\mathrm{U}\left(\mathrm{y}_{\mathrm{i}}, \theta_{\mathrm{i}}\right)>\mathrm{u}\left(\mathrm{C}_{\mathrm{B}}, \theta_{\mathrm{i}}\right) \geq \mathrm{u}\left(\mathrm{C}_{\mathrm{A}}, \theta_{\mathrm{i}}\right)$

$\gamma_{1} \mathrm{~V}\left(\mathrm{y}_{1}\right)+\gamma_{2} \mathrm{~V}\left(\mathrm{y}_{2}\right) \geq \gamma_{1} \mathrm{v}\left(\mathrm{C}_{\mathrm{B}}, \theta_{1}\right)+\gamma_{2} \mathrm{v}\left(\mathrm{C}_{\mathrm{B}}, \theta_{2}\right)-\varepsilon>\gamma_{1} \mathrm{x}_{\mathrm{B}}\left(\theta_{1}\right) \mathrm{v}\left(\mathrm{C}_{\mathrm{B}}, \theta_{1}\right)+\gamma_{2} \mathrm{x}_{\mathrm{B}}\left(\theta_{2}\right)$

$\mathrm{v}\left(\mathrm{C}_{\mathrm{B}}, \theta_{2}\right)$ dès lors que $\mathrm{x}_{\mathrm{B}}\left(\theta_{1}\right)<1$ et $\mathrm{v}\left(\mathrm{C}_{\mathrm{B}}, \theta_{1}\right)>0$.

Donc $B$ ne peut proposer $C_{B}$ à l'équilibre.

b) $\mathrm{x}_{\mathrm{B}}\left(\theta_{1}\right) \mathrm{v}\left(\mathrm{C}_{\mathrm{B}}, \theta_{1}\right)=0$. On a de plus $\mathrm{x}_{\mathrm{B}}\left(\theta_{2}\right)>0$ et $\mathrm{u}\left(\mathrm{C}_{\mathrm{B}}, \theta_{2}\right)<\mathrm{u}^{*}\left(\theta_{2}\right)$, on est dans le cas i. 


\section{Conclusion:}

Si un contrat $C$ est choisi par un agent de type $\theta_{\mathbf{i}}$, alors il vérifie

$\mathrm{u}\left(\mathrm{C}, \theta_{\mathbf{i}}\right) \geq \mathrm{u}^{*}\left(\theta_{\mathbf{i}}\right)$

$\mathrm{v}\left(\mathrm{C}, \theta_{\mathrm{i}}\right) \geq \mathrm{v}^{*}\left(\theta_{\mathrm{i}}\right)$,

par conséquent, on est sûr que l'échange choisi par un agent de type $\theta_{i}$ à l'équilibre est $\mathrm{y}^{*}\left(\theta_{\mathbf{i}}\right)$.

QED

\section{ANNEXE 3: Un exemple}

Soit un modèle principal agent dans lequel l'échange est un couple $(a, t)$ où a désigne une action par exemple et $t$ un transfert observable. Pour un niveau donné de l'échange, si le type de l'agent est $\theta$, le bien-être de chacune des parties s'écrit:

$$
\begin{aligned}
& \mathrm{U}(\mathrm{a}, \mathrm{t}, \theta)=\mathrm{t}-\theta^{\alpha} \mathrm{a}^{2} / 2 \\
& \mathrm{~V}(\mathrm{a}, \mathrm{t}, \theta)=\theta^{\beta} \mathrm{a}-\mathrm{t}
\end{aligned}
$$

Supposons que l'action a n'est pas observable, ou n'est pas vérifiable. Seul un signal z de cette action est observé, et l'on suppose que, quelque soit le type de l'agent $\theta$, il existe une relation entre le type de l'agent, l'action choisie par cet agent et le signal observé, relation décrite par l'équation:

$$
\mathrm{a}=\theta^{1} \mathrm{z}
$$

Si le principal connaît le type de l'agent, observer z équivaut à observer a.

Un contrat entre le principal et l'agent ne peut comporter que des variables observables. L'échange $(a, t)$ est par ailleurs équivalent à l'échange $(z, t)$ et le bien-être des deux parties contractantes peut s'écrire en fonction de l'échange $(z, t)$ de la façon suivante, si le type de l'agent est $\theta$ :

$$
\begin{aligned}
& \text { Le principal obtient: } \theta^{v} z-t \text { avec } v=\beta+1 \\
& \text { L'agent obtient: } t-\theta^{a} z^{2} / 2 \text { avec } a=\alpha+21
\end{aligned}
$$

On note $\Delta=\theta_{1} / \theta_{2}$ et on suppose que $\Delta^{v}>1$.

Imaginons que l'on ait deux structures d'information sur l'action a possible:

La structure 1 est décrite par une relation entre $z$, a et $\theta: a=\theta^{1} z$

La structure 2 est décrite par une relation entre z,a et $\theta: a=\theta g_{z}$

En utilisant la définition proposée en section 4 , la structure 1 est plus précise que la structure 2 si: $-\beta \leq 1<$ g ou $-\beta \geq 1>$ g

En effet, soit:

$\theta_{\mathrm{i}^{1}} \mathrm{z}=\theta_{\mathrm{i}} \mathrm{g} \mathrm{z}^{\prime}$

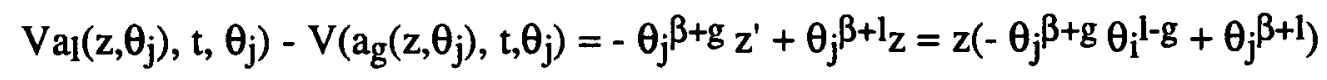


Cette expression est du signe de $1-\left(\theta_{i} / \theta_{j}\right)^{1-g}$

On a de plus

$\mathrm{V}\left(\mathrm{a}_{\mathrm{g}}\left(\mathrm{z}, \theta_{\mathrm{j}}\right), \mathrm{t}, \theta_{\mathrm{j}}\right)-\mathrm{V}\left(\mathrm{a}_{\mathrm{g}}\left(\mathrm{z}, \theta_{\mathrm{i}}\right), \mathrm{t}, \theta_{\mathrm{i}}\right)$ est du signe de $1-\left(\theta_{\mathrm{j}} / \theta_{\mathrm{j}}\right)^{\beta+\mathrm{g}}$

$V\left(a_{1}\left(z, \theta_{j}\right), t, \theta_{j}\right)-V\left(a_{1}\left(z, \theta_{i}\right), t, \theta_{i}\right)$ est du signe de $1-\left(\theta_{i} / \theta_{j}\right)^{\beta+1}$

\section{DETERMINATION DU CONTRAT RS:}

Le contrat de RS est la solution du programme suivant, où l'on cherche $\left(\mathrm{z}_{1}, \mathrm{t}_{1}\right)$ et $\left(\mathrm{z}_{2}, \mathrm{t}_{2}\right)$ :

$$
\begin{aligned}
& \operatorname{Max} \lambda_{1}\left\{t_{1}-\theta_{1}^{a} z_{1}^{2} / 2\right\}+\lambda_{2}\left\{t_{2}-\theta_{2}^{a} z_{2}^{2} / 2\right\} \\
& \text { sc } \\
& t_{1}-\theta_{1}^{a} z_{1}^{2} / 2 \geq t_{2}-\theta_{1}^{a} z_{2}^{2} / 2 \\
& \mathrm{t}_{2}-\theta_{2} \mathrm{a}_{2} \mathrm{z}^{2} / 2 \geq \mathrm{t}_{1}-\theta_{2} \mathrm{a}_{1} \mathrm{z}_{1}^{2} / 2 \\
& \theta_{1} \mathbf{v}_{1}-\mathrm{t}_{1} \geq 0 \\
& \theta_{2} \mathrm{v}_{2}-\mathrm{t}_{2} \geq 0
\end{aligned}
$$

Les deux dernières contraintes sont actives, ce qui nous permet d'écrire: $t_{i}=\theta_{i} \mathbf{v} z_{i}, i=$ 1,2 et le programme devient

$$
\begin{aligned}
& \text { sc } \\
& \theta_{1}{ }^{v} z_{1}-\theta_{1}{ }^{a} z_{1}{ }^{2} / 2 \geq \theta_{2}{ }^{v} z_{2}-\theta_{1}{ }^{a} z_{2}{ }^{2} / 2 \\
& \theta_{2} z_{2}-\theta_{2} z_{2}{ }^{2} / 2 \geq \theta_{1} z_{1}-\theta_{2} z_{1}{ }^{2} / 2
\end{aligned}
$$$$
\operatorname{Max} \lambda_{1}\left\{\theta_{1} \mathrm{v}_{1}-\theta_{1}^{\mathrm{a}} \mathrm{z}_{1}^{2} / 2\right\}+\lambda_{2}\left\{\theta_{2} \mathrm{v}_{2}-\theta_{2}^{\mathrm{a}} \mathrm{z}_{2}^{2} / 2\right\}
$$

Les deux inégalités impliquent que:

$$
\left(1-\Delta^{\mathrm{a}}\right)\left(\mathrm{z}_{1}-\mathrm{z}_{2}\right) \geq 0
$$

1) Le contrat RS est la solution d'information parfaite, les contraintes d'incitation ne sont pas actives. On a :

$$
z_{i}=\theta_{i} \mathrm{v}-\mathrm{a}, \mathrm{i}=1,2
$$

si les paramètres du problème satisfont:

$$
\begin{aligned}
& \theta_{1}^{2 v-a} / 2 \geq \theta_{2}^{2 v-a}-\theta_{1}{ }^{a} \theta_{2} 2 v-2 a / 2 \\
& \theta_{2}{ }^{2 v-a} / 2 \geq \theta_{1}{ }^{2 v-a}-\theta_{2}{ }^{a} \theta_{1}^{2 v-2 a} / 2
\end{aligned}
$$

Ces inégalités peuvent s'écrire:

$$
\begin{aligned}
& \left(\Delta^{2 \mathrm{v}-\mathrm{a}}-1\right)^{2}+\Delta^{2 \mathrm{v}}-1 \geq 0 \\
& \left(\Delta^{\mathrm{v}-\mathrm{a}}-\Delta^{\mathrm{v}}\right)^{2}+1-\Delta^{2 \mathrm{v}} \geq 0
\end{aligned}
$$

La première de ces inégalités est toujours satisfaite puisque $\Delta^{\mathbf{v}} \geq 1$. 


\section{2) La solution d'information parfaite n'est pas possible:}

$$
\left(\Delta^{\mathrm{v}-\mathrm{a}}-\Delta^{\mathrm{v}}\right)^{2}+1-\Delta^{2 \mathrm{v}}<0
$$

Quand le contrat d'information parfaite n'est pas possible, l'une au l'autre des deux contraintes d'incitations est active. La seconde contrainte s'écrit alors

$$
\left(1-\Delta^{a}\right)\left(z_{1}-z_{2}\right) \geq 0
$$

Remarquons que $\mathrm{z}_{1}{ }^{\mathrm{RS}}$ et $\mathrm{z}_{2}{ }^{\mathrm{RS}}$ ne sont pas tous les deux différents de leurs valeurs de premier rang soit respectivement $\theta_{1}{ }^{\mathrm{v}-\mathrm{a}}$ et $\theta_{2}^{\mathrm{v}-\mathrm{a}}$.

En effet, les conditions nécessaires du premier ordre du programme peuvent être écrites, en notant $w_{1}$ le multiplicateur de la première contrainte et $w_{2}$ celui de la seconde contrainte:

$$
\begin{aligned}
& \left(\lambda_{1}+w_{1}\right)\left\{\theta_{1} v-\theta_{1} \mathrm{a} z_{1}\right\}-w_{2}\left\{\theta_{1} v-\theta_{2}^{a} z_{1}\right\}=0 \\
& \left(\lambda_{2}+w_{2}\right)\left\{\theta_{2} v-\theta_{2} z_{2}\right\}-w_{1}\left\{\theta_{2} v-\theta_{1} z_{2}\right\}=0
\end{aligned}
$$

Pour que les solutions soient toutes les deux différentes du premier rang, il est donc nécessaire que les deux contraintes soient actives. Soit:

$$
\begin{aligned}
& \theta_{1}{ }^{\mathrm{v}} z_{1}{ }^{\mathrm{RS}}-\theta_{1}{ }^{\mathrm{a}} \mathrm{z}_{1}{ }^{\mathrm{RS}} / 2=\theta_{2} \mathrm{v} z_{2}{ }^{\mathrm{RS}}-\theta_{1}{ }^{\mathrm{a}} \mathrm{z}_{2} \mathrm{RS} 2 / 2 \\
& \theta_{2}{ }^{\mathrm{v}} z^{\mathrm{RS}}-\theta_{2}^{\mathrm{a}} \mathrm{z}_{2}^{\mathrm{RS}} / 2=\theta_{1}{ }^{\mathrm{v}} \mathrm{z}_{1}^{\mathrm{RS}}-\theta_{2}^{\mathrm{a}} \mathrm{z}_{1}^{\mathrm{RS} 2 / 2}
\end{aligned}
$$

On a donc deux cas possibles:

. La premiere contrainte est active mais pas la seconde:

$z_{1}{ }^{R S}=\theta_{1}{ }^{\mathrm{v}-a}$ et $z_{2}{ }^{R S}$ est défini par: $\theta_{1}{ }^{v} z_{1}{ }^{R S}-\theta_{1}{ }^{a} z_{1}{ }^{R S} 2 / 2=\theta_{2}{ }^{v} z^{R S}-\theta_{1}{ }^{a} z_{2}{ }^{R S} 2 / 2$ avec $\left(1-\Delta^{a}\right)\left(z_{1}{ }^{R S}-z_{2}{ }^{R S}\right) \geq 0$

Cette première solution est impossible, la première équation n'admet aucune racine en $\mathrm{z}_{2}$, son discriminant étant du signe de $1-\Delta^{\mathrm{v}}<0$.

. La seconde contrainte est active mais pas la première: $z_{2}{ }^{R S}=\theta_{2}{ }^{v-a}$ et $z_{1}{ }^{R S}$ est défini par: $\theta_{2}{ }^{v} z_{2}^{R S}-\theta_{2}{ }^{a} z_{2}^{R S} / 2=\theta_{1}{ }^{v} z_{1}^{R S}-\theta_{2} z_{1}^{R S 2} / 2$

$\operatorname{avec}\left(1-\Delta^{\mathrm{a}}\right)\left(\mathrm{z}_{1}^{\mathrm{RS}}-\mathrm{z}_{2}^{\mathrm{RS}}\right) \geq 0$

On a alors

$$
\begin{aligned}
& \mathrm{z}^{\mathrm{RS}}{ }_{1}=\theta_{2}{ }^{\mathrm{v}-\mathrm{a}}\left\{\Delta^{\mathrm{v}}+\mathrm{h}\left(\Delta^{2 \mathrm{v}}-1\right)^{1 / 2}\right\} \text { avec } \mathrm{h}=1 \text { si } \Delta^{\mathrm{a}}<1 \text { et } \mathrm{h}=-1 \text { si } \Delta^{\mathrm{a}}>1 \\
& \mathrm{u}_{1} \mathrm{RS}=\mathrm{u}_{2} \mathrm{RS}+\left(\theta_{2}^{\mathrm{a}}-\theta_{1}^{\mathrm{a}}\right) \mathrm{z}_{1}^{\mathrm{RS} 2 / 2}
\end{aligned}
$$

Le contrat RS:
A) $\Delta^{2 \mathrm{v}-\mathrm{a}}+\Delta^{\mathrm{a}}-2 \geq 0$ et $1-2 \Delta^{2 \mathrm{v}-\mathrm{a}}+\Delta^{2 \mathrm{v}-2 \mathrm{a}} \geq 0$

La solution est la solution d'information parfaite.

B) $\Delta^{2 \mathrm{v}-\mathrm{a}}+\Delta^{\mathrm{a}}-2 \geq 0$ et $1-2 \Delta^{2 \mathrm{v}-\mathrm{a}}+\Delta^{2 \mathrm{v}-2 \mathrm{a}}<0$

$$
\mathrm{z}_{2}^{\mathrm{RS}}=\theta_{2}{ }^{\mathrm{v}-\mathrm{a}}
$$




$$
\begin{aligned}
& \mathrm{z}_{1}^{\mathrm{RS}}=\theta_{1}{ }^{\mathrm{v}-\mathrm{a}}\left\{(\Delta)^{\mathrm{v}}+\mathrm{h}\left[(\Delta)^{2 \mathrm{v}}-1\right]^{1 / 2}\right\} \text { avec si } 1 \geq \Delta^{\mathrm{a}}, \mathrm{h}=1 \text { si } 1<\Delta^{\mathrm{a}}, \mathrm{h}=-1 \\
& \mathrm{u}^{\mathrm{RS}}{ }_{2}=\theta_{2}{ }^{2 \mathrm{v}-\mathrm{a}} / 2 \\
& \mathrm{u}_{1} \mathrm{RS}=\mathrm{u}_{2} \mathrm{RS}+\left(\theta_{2}{ }^{\mathrm{a}}-\theta_{1} \mathrm{a}\right) \mathrm{z}_{1} \mathrm{RS} 2 / 2
\end{aligned}
$$

\section{EXISTENCE DE L'EQUILIBRE RS:}

Le contrat RS est un équilibre s'il n'existe aucune proposition qui vérifie:

$$
\begin{aligned}
& t_{i}-\theta_{i}^{a} z_{i}^{2} / 2 \geq u_{i}^{R S}, i=1,2 \\
& t_{1}-\theta_{1}^{a} z_{1}^{2} / 2 \geq t_{2}-\theta_{1}^{a} z_{2}^{2} / 2 \\
& t_{2}-\theta_{2}^{a} z_{2}^{2} / 2 \geq t_{1}-\theta_{2}^{a} z_{1}{ }^{2} / 2 \\
& \gamma_{1}\left(\theta_{1} v_{1}-t_{1}\right)+\gamma_{2}\left(\theta_{2} z_{2}-t_{2}\right) \geq 0
\end{aligned}
$$

Autrement dit si le contrat RS est solution du programme:

$$
\begin{aligned}
& \operatorname{Max} \gamma_{1}\left(\theta_{1} \mathrm{v}_{1}-\mathrm{t}_{1}\right)+\gamma_{2}\left(\theta_{2} \mathrm{v}_{2}-\mathrm{t}_{2}\right) \\
& \mathrm{t}_{1}-\theta_{1}^{\mathrm{a}} \mathrm{z}_{1}{ }^{2} / 2 \geq \mathrm{t}_{2}-\theta_{1} \mathrm{a}_{2}{ }^{2} / 2\left(\mathrm{w}_{1}\right) \\
& \mathrm{t}_{2}-\theta_{2}^{\mathrm{a}} \mathrm{z}_{2}^{2} / 2 \geq \mathrm{t}_{1}-\theta_{2} \mathrm{a}_{1}{ }^{2} / 2\left(\mathrm{w}_{2}\right) \\
& \mathrm{t}_{1}-\theta_{1}^{\mathrm{a}} \mathrm{z}_{1}{ }^{2} / 2 \geq \mathrm{u}_{1} \mathrm{RS}\left(\mathrm{w}_{3}\right) \\
& \mathrm{t}_{2}-\theta_{2}^{\mathrm{a}} \mathrm{z}_{2}{ }^{2} / 2 \geq \mathrm{u}_{2} \mathrm{RS}\left(\mathrm{w}_{4}\right)
\end{aligned}
$$

Posons $u_{1}=t_{1}-\theta_{1}^{a} z_{1}^{2} / 2$ et $u_{2}=t_{2}-\theta_{2}^{a} z_{2}^{2} / 2$, le programme devient, $P$ :

$$
\begin{aligned}
\operatorname{Max} & \gamma_{1}\left\{\theta_{1}{ }^{v} z_{1}-\theta_{1}{ }^{a} z_{1}^{2} / 2-u_{1}\right\}+\gamma_{2}\left\{\theta_{2}^{v} z_{2}-\theta_{2}^{a} z_{2}^{2} / 2-u_{2}\right\} \\
& u_{1}-u_{2} \geq\left(\theta_{2}{ }^{a}-\theta_{1}^{a}\right) z_{2}^{2} / 2 \\
& u_{1}-u_{2} \leq\left(\theta_{2} a-\theta_{1}^{a}\right) z_{1}^{2} / 2 \\
& u_{1} \geq u_{1} R S \\
& u_{2} \geq u_{2} R S
\end{aligned}
$$

Remarquons dans un premier temps que le contrat $\mathrm{RS}$ est solution du programme $\mathrm{P}$, ce qui nous garantit que le bien-être engendré par sa solution est positif. De plus, si les deux dernières contraintes sont actives, alors la solution de $\mathrm{P}$ est le contrat RS.

Dès lors, il existe un contrat différent du contrat de RS qui détruit l'équilibre du jeu étudié si l'une des deux dernières contraintes n'est pas active, c'est à dire si la solution du programme $P_{1}$ vérifie $u_{2} \geq u_{2} R S$ ou si la solution du programme $P_{2}$ vérifie $u_{1} \geq u_{1} R S$, où $\mathrm{P}_{\mathrm{i}}, \mathrm{i}=1,2$, désigne le programme modifié, où l'on cherche $\mathrm{z}_{1}, \mathrm{u}_{1}, \mathrm{z}_{2}$, et $\mathrm{u}_{2}$ : 


$$
\begin{aligned}
\operatorname{Max} & \gamma_{1}\left\{\theta_{1}{ }^{v} z_{1}-\theta_{1} a z_{1}^{2} / 2-u_{1}\right\}+\gamma_{2}\left\{\theta_{2}{ }^{v} z_{2}-\theta_{2}{ }^{a} z_{2}^{2} / 2-u_{2}\right\} \\
& u_{1}-u_{2} \geq\left(\theta_{2}{ }^{a}-\theta_{1}^{a}\right) z_{2}^{2} / 2 \\
& u_{1}-u_{2} \leq\left(\theta_{2}^{a}-\theta_{1}^{a}\right) z_{1}^{2} / 2 \\
& u_{i} \geq u_{i} R S
\end{aligned}
$$

Le programme $P_{1}$ a une solution très simple qui est donnée par:

$u_{1}=u_{1} R S$ et $u_{2}=u_{1}-\left(\theta_{2}{ }^{a}-\theta_{1}^{a}\right) z_{1}^{2} / 2$

si $\Delta^{\mathrm{a}} \leq \gamma_{2}$ ou $\left.\Delta^{\mathrm{a}} \in\right] 1 ; \gamma_{1} \Delta^{\mathrm{v}}+\gamma_{2}\left[, \mathrm{z}_{1}=\mathrm{z}_{2}=\frac{\gamma_{1} \theta_{1}^{\mathrm{v}}+\gamma_{2} \theta_{2} \mathrm{v}}{\theta_{1}^{\mathrm{a}}}\right.$

si $\left.\Delta_{\mathrm{a}} \in\right] \gamma_{2} ; 1\left[\right.$ ou $\Delta^{\mathrm{a}}>\gamma_{1} \Delta^{\mathrm{v}}+\gamma_{2}, \mathrm{z}_{2}=\theta_{2}^{\mathrm{v}-\mathrm{a}}$ et $\mathrm{z}_{1}=\frac{\gamma_{1} \theta_{1} \mathrm{v}}{\theta_{1}^{\mathrm{a}}-\gamma_{2} \theta_{2}^{\mathrm{a}}}$

Et le contrat RS n'est pas un équilibre si $\left(1-\Delta^{\mathrm{a}}\right)\left(\mathrm{z}_{1} \mathrm{RS}-\mathrm{z}_{1}\right)>0$

Le programme $\mathrm{P}_{2}$ a lui aussi une solution simple, donnée par:

$$
\begin{aligned}
& u_{1}=u_{2}+\left(\theta_{2}^{a}-\theta_{1}^{a}\right) z_{2} 2 / 2 \text { et } u_{2}=u_{2} R S \\
& \text { si } \Delta^{\mathrm{a}}<1 \text { ou } \Delta^{\mathrm{a}} \in\left[\frac{\Delta^{\mathrm{v}}}{\gamma_{1} \Delta^{\mathrm{v}}+\gamma_{2}} ; 1 / \gamma_{1}\left[, \mathrm{z}_{1}=\theta_{1}^{\mathrm{v}-\mathrm{a}} \text { et } \mathrm{z}_{2}=\frac{\gamma_{2} \theta_{2}^{\mathrm{v}}}{\theta_{2}^{\mathrm{a}}-\gamma_{1} \theta_{1}^{\mathrm{a}}}\right.\right. \\
& \text { si } \left.\left.\Delta^{\mathrm{a}} \in\right] 1 ; \frac{\Delta^{\mathrm{v}}}{\gamma_{1} \Delta^{\mathrm{v}}+\gamma_{2}}\right] \text { ou } \Delta^{\mathrm{a}}>1 / \gamma_{1} \text {, alors } \mathrm{z}_{1}=\mathrm{z}_{2}=\frac{\gamma_{1} \theta_{1} \mathrm{v}+\gamma_{2} \theta_{2} \mathrm{v}}{\theta_{2}^{\mathrm{a}}}
\end{aligned}
$$

Le contrat de RS n'est pas alors un équilibre si

$\left(1-\Delta^{\mathrm{a}}\right)\left(\mathrm{z}_{2}-\mathrm{z}_{1}^{\mathrm{RS}}\right)>0$

On obtient alors aisément, si:

$$
\Delta^{\mathrm{a}} \leq \frac{\Delta^{\mathrm{v}}}{\Delta^{\mathrm{v}}+\left(\Delta^{2 \mathrm{v}}-1\right)^{1 / 2}}
$$

le contrat de RS est le premier rang

$$
\frac{\Delta^{v}}{\Delta^{v}+\left(\Delta^{2 v}-1\right)^{1 / 2}}<\Delta^{\mathrm{a}}<\frac{\Delta^{\mathrm{v}}+\gamma_{2}\left(\Delta^{2 v}-1\right)^{1 / 2}}{\Delta^{\mathrm{v}}+\left(\Delta^{2 \mathrm{v}}-1\right)^{1 / 2}}
$$

le contrat RS est le second rang, c'est un équilibre

$$
\frac{\Delta^{v}+\gamma_{2}\left(\Delta^{2 v}-1\right)^{1 / 2}}{\Delta^{v}+\left(\Delta^{2 v}-1\right)^{1 / 2}} \leq \Delta^{\mathrm{a}} \leq \frac{\Delta^{\mathrm{v}}-\gamma_{2}\left(\Delta^{2 v}-1\right)^{1 / 2}}{\Delta^{v}-\left(\Delta^{2 v}-1\right)^{1 / 2}}
$$

RS n'est pas un équilibre

$$
\frac{\Delta^{\mathrm{v}}-\gamma_{2}\left(\Delta^{2 v}-1\right)^{1 / 2}}{\Delta^{\mathrm{v}}-\left(\Delta^{2 v}-1\right)^{1 / 2}}<\Delta^{\mathrm{a}}<\frac{\Delta^{\mathrm{v}}}{\Delta^{\mathrm{v}}-\left(\Delta^{2 \mathrm{v}}-1\right)^{1 / 2}} \text {, RS est un équilibre , c'est }
$$

le second rang

$\frac{\Delta^{\mathrm{v}}}{\Delta^{\mathrm{v}}-\left(\Delta^{2 \mathrm{v}}-1\right)^{1 / 2}} \leq \Delta^{\mathrm{a}}$

RS est le premier rang 


\section{CONTRE-EXEMPLE:}

Supposons que $\Delta=2, \beta=0, \mathrm{~g}=1$ et $\mathrm{l}=1 / 2, \alpha=0,1$.

$\mathrm{g}>1>0$, la structure d'information 2 (g) est donc moins précise que la structure d'information 1 (l)

Pour la structure $2, \mathrm{v}=1$ et $\mathrm{a}=2,1, \mathrm{RS}$ est un équilibre: on a

$\frac{\Delta^{v}}{\Delta^{\mathrm{a}}\left\{\Delta^{\mathrm{v}}-\left(\Delta^{2 v}-1\right)^{1 / 2}\right\}}=\frac{2}{2^{2,1}\left\{2-(3)^{1 / 2}\right\}}>1$

$\frac{\Delta^{v}-\gamma_{2}\left(\Delta^{2 v}-1\right)^{1 / 2}}{\Delta^{\mathrm{a}}\left\{\Delta^{\mathrm{v}}-\left(\Delta^{2 v}-1\right)^{1 / 2}\right\}}=\frac{2-1 / 2(3)^{1 / 2}}{2^{2,1}\left\{2-(3)^{1 / 2}\right\}}<1$

Mais RS n'est pas un équilibre pour la structure d'information 1 où $1=1 / 2$, $(\mathrm{v}=1 / 2$ et a $=1,1)$ car dans ce cas:

$\left.\frac{\Delta^{v}+\gamma_{2}\left(\Delta^{2 v}-1\right)^{1 / 2}}{\Delta^{\mathrm{a}}\left\{\Delta^{\mathrm{v}}+\left(\Delta^{2 v}-1\right)^{1 / 2}\right.}\right\}=\frac{2^{1 / 2}+1 / 2}{2^{1,1}\left(2^{1 / 2}+1\right\}}<1$
$\frac{\Delta^{\mathrm{v}}-\gamma_{2}\left(\Delta^{2 v}-1\right)^{1 / 2}}{\Delta^{\mathrm{a}}\left\{\Delta^{\mathrm{v}}-\left(\Delta^{2 v}-1\right)^{1 / 2}\right\}}=\frac{2^{1 / 2}-1 / 2}{2^{1,1}\left\{2^{1 / 2}-1\right\}}>1$ 


\section{Références:}

CROCKER K. et SNOW A. (1985): "The Efficiency of Competitive Equilibria in Insurance Markets with Asymmetric Information" Journal of Public Economics, 26, 207. 219

GROSSMAN, H.I(1979): " Adverse selection, Dissembling, and Competitive Equilibrium" Bell Journal of Economics, 10, 336-343

HELLWIG M.F. (1987): " Some recent Developements in the Theory of Competition in Markets with Adverse Selection" European Economic Review, 31, 319-325

HENRIET et ROCHET 1987 : "Micro-économie de l'assurance"

MASKINS E. et TIROLE J. (1990): " The Principal-Agent Relationship with an Informed Principal: the case of Private Values" Econometrica, 58, 379-409

MIAZAKY, H ( 1977): " The Rate Race and Internal Labour Markets" Bell Journal of Economics 8, 394-418

ROSENTHAL R.W. et WEISS A. (1984): " Mixed Strategy Equilibrium in a Market with Asymetric Information "Review of Economic Studies, LI, 333-342

ROTHSCHILD M. et STIGLITZ J. (1976): "Equilibrium in Competitive Insurance Markets: An Essay on the Economics of Imperfect Information" Quaterly Journal of Economics, 90, 629-650

ROTHSCHILD M. et STIGLITZ J. (1970): "Increasing risk I: A definition " Journal of Economic Theory, 2, 315-329

RILEY J. (1979) "Informational Equilibrium" Econometrica, 331-359

DASGUPTA, P et MASKINS, E (1986) : "The Existence of Equilibrium in Disontinous Economic Games, II: Aplications", Review of Economic Studies, 53, 27-41

WILSON C. (1977): "A model of Insurance Market with incomplete Information" Journal of Economic Theory, 167-207 
WILSON C. (1979): " Equilibrium and Adverse Selection" American Economic Review, 313-317

WILSON C. (1980):" The Nature of Equilibrium in Markets with Adverse Selection" Bell Journal of Economics, 108-130 